Acta Crystallographica Section E

Structure Reports

Online

ISSN 1600-5368

\section{Dicarbonyl $\left\{3,3^{\prime}\right.$-di-tert-butyl-5, $5^{\prime}$-di- methoxy-2,2'-bis[(4,4,5,5-tetraphenyl- 1,3,2-dioxaphospholan-2-yl)oxy- $\mathrm{k} P$ ]- biphenyl\}hydridorhodium(I) diethyl ether monosolvate}

\section{Detlef Selent,* Anke Spannenberg and Armin Börner}

Leibniz-Institut für Katalyse e. V. an der Universität Rostock, Albert-Einstein-Strasse 29a, 18059 Rostock, Germany

Correspondence e-mail: detlef.selent@catalysis.de

Received 16 March 2012; accepted 19 March 2012

Key indicators: single-crystal X-ray study; $T=200 \mathrm{~K}$; mean $\sigma(\mathrm{C}-\mathrm{C})=0.003 \AA$; $R$ factor $=0.028 ; w R$ factor $=0.063 ;$ data-to-parameter ratio $=19.5$.

In the title compound, $\left[\mathrm{Rh}\left(\mathrm{C}_{74} \mathrm{H}_{68} \mathrm{O}_{8} \mathrm{P}_{2}\right) \mathrm{H}(\mathrm{CO})_{2}\right] \cdot \mathrm{C}_{4} \mathrm{H}_{10} \mathrm{O}$, the $\mathrm{C}_{2} \mathrm{HP}_{2}$ coordination set at the $\mathrm{Rh}^{\mathrm{I}}$ ion is arranged in a distorted trigonal-planar geometry with one $\mathrm{P}$ atom of the diphosphite molecule and the $\mathrm{H}$ atom adopting the axial coordination sites.

\section{Related literature}

For another crystal structure of a dicarbonyl hydrido complex of rhodium(I), see: Van Rooy et al. (1995, 1996). The title compound has recently been studied in solution, see: Selent et al. (2011). Structural information on this labile compound class is usually obtained by spectroscopy, see, for example: Dieleman et al. (2001); Axet et al. (2007).

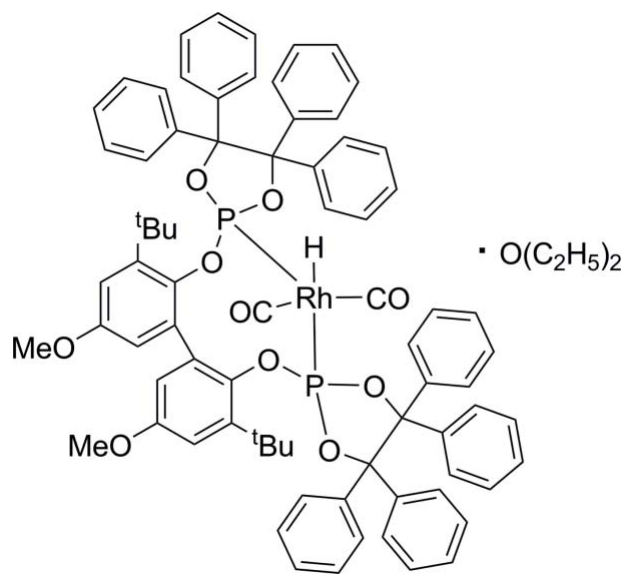

\section{Experimental}

Crystal data

$\left[\mathrm{Rh}\left(\mathrm{C}_{74} \mathrm{H}_{68} \mathrm{O}_{8} \mathrm{P}_{2}\right) \mathrm{H}(\mathrm{CO})_{2}\right] \cdot \mathrm{C}_{4} \mathrm{H}_{10} \mathrm{O}$

$M_{r}=1381.28$

Monoclinic, $P 2_{1}$

$a=11.1489$ (2) ^

$b=25.8458$ (4) $\AA$

$c=12.5941(3) \AA$

$\beta=110.263(2)^{\circ}$

$V=3404.43(11) \AA^{3}$

$Z=2$

Mo $K \alpha$ radiation

$\mu=0.36 \mathrm{~mm}^{-1}$

$T=200 \mathrm{~K}$

$0.40 \times 0.35 \times 0.15 \mathrm{~mm}$

\section{Data collection}

Stoe IPDS II diffractometer

Absorption correction: numerical

$(X$-SHAPE and $X$-RED32;

Stoe \& Cie, 2005)

$T_{\min }=0.835, T_{\max }=0.959$

Refinement

$R\left[F^{2}>2 \sigma\left(F^{2}\right)\right]=0.028$

$w R\left(F^{2}\right)=0.063$

$S=0.94$

16262 reflections

834 parameters

35 restraints
59171 measured reflections 16262 independent reflections 14238 reflections with $I>2 \sigma(I)$ $R_{\text {int }}=0.026$

Data collection: $X$-AREA (Stoe \& Cie, 2005); cell refinement: $X$ $A R E A$; data reduction: $X$ - $A R E A$; $\operatorname{program}(\mathrm{s})$ used to solve structure: SHELXS97 (Sheldrick, 2008); program(s) used to refine structure: SHELXL97 (Sheldrick, 2008); molecular graphics: XP in SHELXTL (Sheldrick, 2008); software used to prepare material for publication: SHELXTL.

Supplementary data and figures for this paper are available from the IUCr electronic archives (Reference: BT5848).

\title{
References
}

Axet, M. R., Benet-Buchholz, J., Claver, C. \& Castillón, S. (2007). Adv. Synth. Catal. 349, 1983-1998.

Dieleman, C. B., Kamer, P. C. J., Reek, J. N. H. \& van Leeuwen, P. W. N. M. (2001). Helv. Chim. Acta, 84, 3269-3280.

Flack, H. D. (1983). Acta Cryst. A39, 876-881.

Selent, D., Franke, R., Kubis, C., Spannenberg, A., Baumann, W., Kreidler, B. \& Börner, A. (2011). Organometallics, 30, 4509-4514.

Sheldrick, G. M. (2008). Acta Cryst. A64, 112-122.

Stoe \& Cie (2005). X-SHAPE, X-RED32 and X-AREA. Stoe \& Cie, Darmstadt, Germany.

Van Rooy, A., Kamer, P. C. J., van Leeuwen, P. W. N. M., Goubitz, K., Fraanje, J., Veldman, N. \& Spek, A. L. (1996). Organometallics, 15, 835-847.

Van Rooy, A., Kamer, P. C. J., van Leeuwen, P. W. N. M., Veldman, N. \& Spek, A. L. (1995). J. Organomet. Chem. 494, C15-C18. 


\title{
supporting information
}

Acta Cryst. (2012). E68, m488 [https://doi.org/10.1107/S1600536812011786]

Dicarbonyl\{3,3'-di-tert-butyl-5, 5'-dimethoxy-2,2'-bis[(4,4,5,5-tetraphenyl-1,3,2dioxaphospholan-2-yl)oxy- $\kappa$ P]biphenyl\}hydridorhodium(I) diethyl ether monosolvate

\author{
Detlef Selent, Anke Spannenberg and Armin Börner
}

\section{S1. Comment}

The reaction of a mixture of dicarbonyl(acetylacetonato- $\kappa O, O^{\prime}$ )rhodium(I) and 3,3'-di-tert-butyl-5,5'-dimethoxy-2,2'-bis[(4,4,5,5-tetraphenyl-1,3,2-dioxaphospholan-2-yl)oxy]biphenyl under an atmosphere of hydrogen and carbon monoxide in toluene affords the elimination of acetylacetone and the formation of the rhodium hydrido complex which, after recrystallization, gives the title compound (figure 1). The distances $\mathrm{Rh} 1-\mathrm{P} 1=2.3045$ (5) $\AA$ and $\mathrm{Rh} 1-\mathrm{P} 2=2.2913$ (5) $\AA$ and the angle $\mathrm{P} 1-\mathrm{Rh} 1-\mathrm{P} 2=109.66(2)^{\circ}$ do significantly differ from those $(\mathrm{Rh} 1-\mathrm{P} 1=2.255$ (3), Rh1—P2 = 2.239 (3) $\left.\AA, \mathrm{P} 1-\mathrm{Rh} 1-\mathrm{P} 2=115.95(9)^{\circ}\right)$ found in the only molecular structure of a similar rhodium complex known to date (Van Rooy et al. 1995, 1996). In the solid state, the phosphorus atoms of the diphosphite molecule adopt formally different axial and equatorial sites of the idealized trigonal bipyramidal geometry around the rhodium center which is in contrast to the bisequatorial arrangement determined for both, the solution and the theoretical gas phase structure (Selent et al., 2011). The hydride could be found from difference Fourier map; the Rh1—H1 distance was refined to 1.42 (3) $\AA$.

\section{S2. Experimental}

A mixture of dicarbonyl(acetylacetonato- $\left.\kappa O, O^{\prime}\right)$ rhodium(I) $(0.5161 \mathrm{~g}, 2 \mathrm{mmol})$ and 3,3'-di-tert-butyl-5,5'-dimethoxy-2,2'bis[(4,4,5,5-tetraphenyl-1,3,2-dioxaphospholan-2-yl)oxy]biphenyl (2.524 g, $2.2 \mathrm{mmol})$ was dissolved in toluene (50 ml). The resulting solution was transferred to an autoclave and then stirred under an atmosphere of carbon monoxide and hydrogen $(1: 1,2.0 \mathrm{MPa})$ at $70^{\circ} \mathrm{C}$ for $2 \mathrm{~h}$. The clear, pale yellow solution obtained after cooling and depressurization was evaporated to dryness in vacuo. The residue was crystallized from diethyl ether to give $2.108 \mathrm{~g}(1.613 \mathrm{mmol}, 81 \%)$ of the

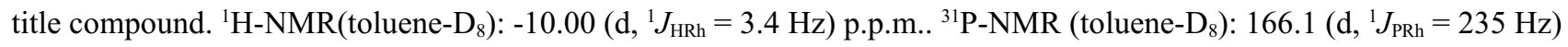
p.p.m.. Elemental analysis (calc. for $\mathrm{C}_{76} \mathrm{H}_{69} \mathrm{O}_{10} \mathrm{P}_{2} \mathrm{Rh}=1307.229 \mathrm{~g} / \mathrm{mol}$ ): C, 69.75 (69.83); H, 5.48 (5.32); P, 4.56 (4.74); Rh, 7.73(7.87) \%. ESI-TOF/HRMS: m/e $1317.3097(\mathrm{M}-\mathrm{CO}+\mathrm{K})^{+}, 1249.34397(M-2 \mathrm{CO})^{+}$. Crystals suitable for X-ray analysis were obtained by recrystallization from diethyl ether.

\section{S3. Refinement}

$\mathrm{H} 1 \mathrm{was}$ found from difference Fourier map and refined freely. All other $\mathrm{H}$ atoms were placed in idealized positions with $\mathrm{d}(\mathrm{C}-\mathrm{H})=0.95 \AA(\mathrm{CH}), 0.99 \AA\left(\mathrm{CH}_{2}\right)$ and $0.98 \AA\left(\mathrm{CH}_{3}\right)$ and refined using a riding model with $U_{\text {iso }}(\mathrm{H})$ fixed at $1.2 U_{\text {eq }}(\mathrm{C})$ for $\mathrm{CH}, \mathrm{CH}_{2}$ and $1.5 \mathrm{U}_{\text {eq }}(\mathrm{C})$ for $\mathrm{CH}_{3}$. The solvent atoms were only isotropically refined. 


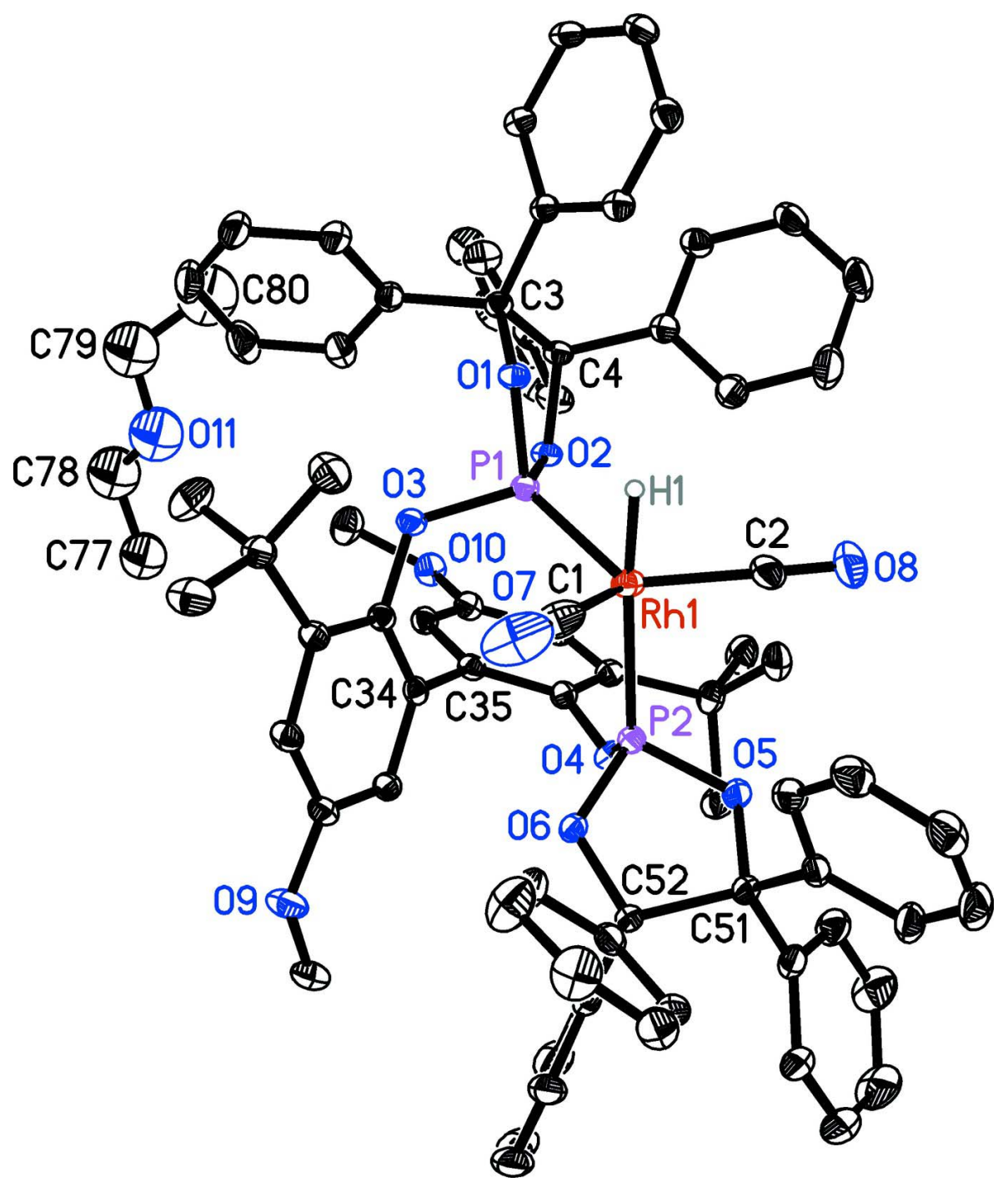

Figure 1

The molecular structure of the title compound; displacement ellipsoids are drawn at the $30 \%$ probability level. Hydrogen atoms except $\mathrm{H} 1$ are omitted for clarity.

Dicarbonyl\{3,3'-di-tert-butyl-5,5'-dimethoxy-2,2'-bis[(4,4,5,5- tetraphenyl-1,3,2-dioxaphospholan-2-yl)oxy$\kappa P]$ biphenyl\}hydridorhodium(I) diethyl ether monosolvate

Crystal data

$\left[\mathrm{Rh}\left(\mathrm{C}_{74} \mathrm{H}_{68} \mathrm{O}_{8} \mathrm{P}_{2}\right) \mathrm{H}(\mathrm{CO})_{2}\right] \cdot \mathrm{C}_{4} \mathrm{H}_{10} \mathrm{O}$

$M_{r}=1381.28$

Monoclinic, $P 2_{1}$

$a=11.1489(2) \AA$

$b=25.8458(4) \AA$

$c=12.5941(3) \AA$

$\beta=110.263(2)^{\circ}$

$V=3404.43(11) \AA^{3}$

$Z=2$
$F(000)=1444$

$D_{\mathrm{x}}=1.347 \mathrm{Mg} \mathrm{m}^{-3}$

Mo $K \alpha$ radiation, $\lambda=0.71073 \AA$

Cell parameters from 15841 reflections

$\theta=1.7-28.4^{\circ}$

$\mu=0.36 \mathrm{~mm}^{-1}$

$T=200 \mathrm{~K}$

Prism, colourless

$0.40 \times 0.35 \times 0.15 \mathrm{~mm}$ 


\section{Data collection}

Stoe IPDS II

diffractometer

Radiation source: fine-focus sealed tube

Graphite monochromator

$\omega$ scans

Absorption correction: numerical

( $X$-SHAPE and $X$-RED32; Stoe \& Cie, 2005)

$T_{\min }=0.835, T_{\max }=0.959$

\section{Refinement}

Refinement on $F^{2}$

Least-squares matrix: full

$R\left[F^{2}>2 \sigma\left(F^{2}\right)\right]=0.028$

$w R\left(F^{2}\right)=0.063$

$S=0.94$

16262 reflections

834 parameters

35 restraints

Primary atom site location: structure-invariant direct methods

Secondary atom site location: difference Fourier map
59171 measured reflections

16262 independent reflections

14238 reflections with $I>2 \sigma(I)$

$R_{\text {int }}=0.026$

$\theta_{\max }=27.9^{\circ}, \theta_{\min }=1.6^{\circ}$

$h=-14 \rightarrow 14$

$k=-34 \rightarrow 34$

$l=-16 \rightarrow 16$

Hydrogen site location: inferred from neighbouring sites

$\mathrm{H}$ atoms treated by a mixture of independent and constrained refinement

$w=1 /\left[\sigma^{2}\left(F_{0}^{2}\right)+(0.0377 P)^{2}\right]$

where $P=\left(F_{\mathrm{o}}{ }^{2}+2 F_{\mathrm{c}}{ }^{2}\right) / 3$

$(\Delta / \sigma)_{\max }=0.001$

$\Delta \rho_{\max }=0.66 \mathrm{e} \AA^{-3}$

$\Delta \rho_{\min }=-0.47$ e $\AA^{-3}$

Absolute structure: Flack (1983), 7944 Friedel pairs

Absolute structure parameter: $-0.026(11)$

\section{Special details}

Geometry. All e.s.d.'s (except the e.s.d. in the dihedral angle between two 1.s. planes) are estimated using the full covariance matrix. The cell e.s.d.'s are taken into account individually in the estimation of e.s.d.'s in distances, angles and torsion angles; correlations between e.s.d.'s in cell parameters are only used when they are defined by crystal symmetry. An approximate (isotropic) treatment of cell e.s.d.'s is used for estimating e.s.d.'s involving 1.s. planes.

Refinement. Refinement of $F^{2}$ against ALL reflections. The weighted $R$-factor $w R$ and goodness of fit $S$ are based on $F^{2}$, conventional $R$-factors $R$ are based on $F$, with $F$ set to zero for negative $F^{2}$. The threshold expression of $F^{2}>\sigma\left(F^{2}\right)$ is used only for calculating $R$-factors(gt) etc. and is not relevant to the choice of reflections for refinement. $R$-factors based on $F^{2}$ are statistically about twice as large as those based on $F$, and $R$ - factors based on ALL data will be even larger.

Fractional atomic coordinates and isotropic or equivalent isotropic displacement parameters $\left(\AA^{2}\right)$

\begin{tabular}{lllll}
\hline & $x$ & $y$ & $z$ & $U_{\text {iso }} / U_{\text {eq }}$ \\
\hline C77 & $1.0804(4)$ & $0.91333(17)$ & $0.5094(3)$ & $0.0878(11)^{*}$ \\
H77A & 1.1508 & 0.9343 & 0.5030 & $0.132^{*}$ \\
H77B & 1.1042 & 0.8767 & 0.5147 & $0.132^{*}$ \\
H77C & 1.0626 & 0.9236 & 0.5774 & $0.132^{*}$ \\
C78 & $0.9699(5)$ & $0.9212(2)$ & $0.4129(4)$ & $0.1113(15)^{*}$ \\
H78A & 0.9490 & 0.9586 & 0.4077 & $0.134^{*}$ \\
H78B & 0.9908 & 0.9120 & 0.3449 & $0.134^{*}$ \\
C79 & $0.7521(5)$ & $0.9016(2)$ & $0.3186(4)$ & $0.1172(17)^{*}$ \\
H79A & 0.7315 & 0.8699 & 0.2715 & $0.141^{*}$ \\
H79B & 0.7635 & 0.9304 & 0.2713 & $0.141^{*}$ \\
C80 & $0.6510(6)$ & $0.9131(3)$ & $0.3584(6)$ & $0.147(2)^{*}$ \\
H80A & 0.5718 & 0.9181 & 0.2938 & $0.221^{*}$ \\
H80B & 0.6711 & 0.9449 & 0.4037 & $0.221^{*}$ \\
H80C & 0.6401 & 0.8845 & 0.4052 & $0.221^{*}$ \\
O11 & $0.8631(3)$ & $0.89420(13)$ & $0.4096(3)$ & $0.1138(10)^{*}$
\end{tabular}




\begin{tabular}{|c|c|c|c|c|}
\hline $\mathrm{C} 1$ & $0.5785(3)$ & $0.53367(10)$ & $0.6920(2)$ & $0.0518(7)$ \\
\hline $\mathrm{C} 2$ & $0.5159(2)$ & $0.59995(12)$ & $0.9031(2)$ & $0.0456(6)$ \\
\hline $\mathrm{C} 3$ & $0.37567(19)$ & $0.72557(7)$ & $0.53935(15)$ & $0.0230(4)$ \\
\hline $\mathrm{C} 4$ & $0.44781(18)$ & $0.75555(7)$ & $0.65556(16)$ & $0.0248(4)$ \\
\hline $\mathrm{C} 5$ & $0.4165(2)$ & $0.73789(8)$ & $0.43790(16)$ & $0.0287(4)$ \\
\hline $\mathrm{C} 6$ & $0.3402(2)$ & $0.72021(9)$ & $0.33200(16)$ & $0.0377(5)$ \\
\hline H6 & 0.2633 & 0.7022 & 0.3239 & $0.045^{*}$ \\
\hline $\mathrm{C} 7$ & $0.3749(2)$ & $0.72856(10)$ & $0.23819(19)$ & $0.0507(7)$ \\
\hline $\mathrm{H} 7$ & 0.3217 & 0.7161 & 0.1664 & $0.061^{*}$ \\
\hline $\mathrm{C} 8$ & $0.4858(2)$ & $0.75483(11)$ & 0.24739 (19) & $0.0573(8)$ \\
\hline $\mathrm{H} 8$ & 0.5071 & 0.7620 & 0.1821 & $0.069^{*}$ \\
\hline C9 & $0.5654(3)$ & $0.77058(10)$ & $0.35312(17)$ & $0.0522(7)$ \\
\hline H9 & 0.6438 & 0.7874 & 0.3610 & $0.063^{*}$ \\
\hline $\mathrm{C} 10$ & 0.5319 (2) & $0.76214(9)$ & $0.44750(19)$ & $0.0375(5)$ \\
\hline $\mathrm{H} 10$ & 0.5879 & 0.7729 & 0.5198 & $0.045^{*}$ \\
\hline $\mathrm{C} 11$ & $0.23051(19)$ & $0.72664(7)$ & $0.50856(15)$ & $0.0242(4)$ \\
\hline $\mathrm{C} 12$ & $0.1685(2)$ & $0.68911(8)$ & $0.54920(17)$ & $0.0297(4)$ \\
\hline H12 & 0.2158 & 0.6610 & 0.5922 & $0.036^{*}$ \\
\hline $\mathrm{C} 13$ & $0.0377(2)$ & $0.69246(9)$ & $0.52739(19)$ & $0.0346(5)$ \\
\hline H13 & -0.0041 & 0.6662 & 0.5544 & $0.042 *$ \\
\hline $\mathrm{C} 14$ & $-0.0318(2)$ & $0.73353(9)$ & $0.46688(18)$ & $0.0347(5)$ \\
\hline H14 & -0.1209 & 0.7360 & 0.4534 & $0.042 *$ \\
\hline $\mathrm{C} 15$ & $0.0287(2)$ & $0.77076(8)$ & $0.42623(17)$ & $0.0338(5)$ \\
\hline H15 & -0.0188 & 0.7992 & 0.3846 & $0.041 *$ \\
\hline $\mathrm{C} 16$ & $0.1582(2)$ & $0.76722(8)$ & $0.44540(16)$ & $0.0289(4)$ \\
\hline H16 & 0.1984 & 0.7928 & 0.4151 & $0.035^{*}$ \\
\hline $\mathrm{C} 17$ & $0.37177(18)$ & $0.75349(6)$ & $0.73659(15)$ & $0.0268(4)$ \\
\hline $\mathrm{C} 18$ & $0.27163(19)$ & $0.78775(8)$ & $0.72410(17)$ & $0.0316(4)$ \\
\hline H18 & 0.2514 & 0.8130 & 0.6657 & $0.038^{*}$ \\
\hline C19 & $0.2012(2)$ & $0.78574(9)$ & $0.79491(17)$ & $0.0406(5)$ \\
\hline H19 & 0.1335 & 0.8096 & 0.7850 & $0.049 *$ \\
\hline $\mathrm{C} 20$ & $0.2282(3)$ & $0.74940(9)$ & $0.8798(2)$ & $0.0542(7)$ \\
\hline $\mathrm{H} 20$ & 0.1775 & 0.7471 & 0.9266 & $0.065^{*}$ \\
\hline $\mathrm{C} 21$ & $0.3298(3)$ & $0.71647(12)$ & $0.8955(2)$ & $0.0654(9)$ \\
\hline $\mathrm{H} 21$ & 0.3513 & 0.6921 & 0.9557 & $0.078^{*}$ \\
\hline $\mathrm{C} 22$ & $0.4007(3)$ & $0.71839(10)$ & 0.82517 (19) & $0.0470(6)$ \\
\hline $\mathrm{H} 22$ & 0.4705 & 0.6953 & 0.8375 & $0.056^{*}$ \\
\hline $\mathrm{C} 23$ & $0.4872(2)$ & $0.81179(7)$ & $0.65172(18)$ & $0.0287(4)$ \\
\hline $\mathrm{C} 24$ & $0.4264(2)$ & $0.84704(7)$ & $0.5669(2)$ & $0.0407(5)$ \\
\hline $\mathrm{H} 24$ & 0.3584 & 0.8356 & 0.5019 & $0.049^{*}$ \\
\hline $\mathrm{C} 25$ & $0.4628(3)$ & $0.89834(8)$ & $0.5752(2)$ & $0.0495(6)$ \\
\hline $\mathrm{H} 25$ & 0.4194 & 0.9218 & 0.5163 & $0.059 *$ \\
\hline $\mathrm{C} 26$ & $0.5618(2)$ & $0.91583(9)$ & $0.66831(19)$ & $0.0506(7)$ \\
\hline $\mathrm{H} 26$ & 0.5870 & 0.9511 & 0.6740 & $0.061 *$ \\
\hline $\mathrm{C} 27$ & $0.6238(2)$ & $0.88133(7)$ & $0.7529(2)$ & $0.0505(7)$ \\
\hline $\mathrm{H} 27$ & 0.6930 & 0.8928 & 0.8170 & $0.061 *$ \\
\hline $\mathrm{C} 28$ & $0.5857(2)$ & $0.83023(8)$ & $0.7449(2)$ & $0.0377(5)$ \\
\hline $\mathrm{H} 28$ & 0.6282 & 0.8071 & 0.8048 & $0.045^{*}$ \\
\hline
\end{tabular}




\begin{tabular}{|c|c|c|c|c|}
\hline C29 & $0.75300(19)$ & $0.63414(7)$ & $0.60830(16)$ & $0.0236(4)$ \\
\hline $\mathrm{C} 30$ & $0.75663(16)$ & $0.59539(10)$ & $0.53180(14)$ & $0.0268(3)$ \\
\hline $\mathrm{C} 31$ & $0.8683(2)$ & $0.56572(7)$ & $0.56195(17)$ & $0.0298(4)$ \\
\hline $\mathrm{H} 31$ & 0.8735 & 0.5385 & 0.5130 & $0.036^{*}$ \\
\hline $\mathrm{C} 32$ & $0.9723(2)$ & $0.57425(8)$ & $0.66043(18)$ & $0.0282(4)$ \\
\hline $\mathrm{C} 33$ & $0.96708(19)$ & $0.61368(7)$ & $0.73284(16)$ & $0.0253(4)$ \\
\hline $\mathrm{H} 33$ & 1.0380 & 0.6202 & 0.7998 & $0.030^{*}$ \\
\hline C34 & $0.85706(19)$ & $0.64382(7)$ & $0.70688(16)$ & $0.0225(4)$ \\
\hline $\mathrm{C} 35$ & $0.85814(18)$ & $0.68962(7)$ & $0.77842(16)$ & $0.0241(4)$ \\
\hline $\mathrm{C} 36$ & 0.85984 (19) & $0.68565(7)$ & $0.88844(16)$ & $0.0240(4)$ \\
\hline C37 & $0.8629(2)$ & $0.72994(8)$ & $0.95425(16)$ & $0.0260(4)$ \\
\hline C38 & $0.8742(2)$ & $0.77697(8)$ & $0.90746(17)$ & 0.0285 \\
\hline $\mathrm{H} 38$ & 0.8807 & 0.8074 & 0.9516 & $0.034 *$ \\
\hline C39 & $0.8764(2)$ & $0.78185(7)$ & $0.79794(18)$ & 0.0277 (4) \\
\hline $\mathrm{C} 40$ & 0.86457 (19) & $0.73841(7)$ & $0.73202(17)$ & $0.0256(4)$ \\
\hline $\mathrm{H} 40$ & 0.8608 & 0.7414 & 0.6557 & $0.031 *$ \\
\hline $\mathrm{C} 41$ & 0.65315 (19) & $0.58828(10)$ & $0.41376(16)$ & $0.0347(5)$ \\
\hline $\mathrm{C} 42$ & $0.6587(4)$ & $0.63533(12)$ & $0.3432(2)$ & $0.0581(8)$ \\
\hline $\mathrm{H} 42 \mathrm{~A}$ & 0.6297 & 0.6659 & 0.3735 & $0.087^{*}$ \\
\hline $\mathrm{H} 42 \mathrm{~B}$ & 0.6031 & 0.6298 & 0.2646 & $0.087^{*}$ \\
\hline $\mathrm{H} 42 \mathrm{C}$ & 0.7468 & 0.6406 & 0.3462 & $0.087^{*}$ \\
\hline $\mathrm{C} 43$ & $0.5178(2)$ & $0.58264(12)$ & $0.4152(2)$ & $0.0536(8)$ \\
\hline $\mathrm{H} 43 \mathrm{~A}$ & 0.4934 & 0.6144 & 0.4453 & $0.080^{*}$ \\
\hline H43B & 0.5143 & 0.5533 & 0.4633 & $0.080^{*}$ \\
\hline $\mathrm{H} 43 \mathrm{C}$ & 0.4585 & 0.5767 & 0.3381 & $0.080^{*}$ \\
\hline $\mathrm{C} 44$ & $0.6792(3)$ & $0.53988(12)$ & $0.3542(2)$ & $0.0529(8)$ \\
\hline $\mathrm{H} 44 \mathrm{~A}$ & 0.6122 & 0.5363 & 0.2797 & $0.079^{*}$ \\
\hline H44B & 0.6794 & 0.5092 & 0.4000 & $0.079^{*}$ \\
\hline $\mathrm{H} 44 \mathrm{C}$ & 0.7625 & 0.5432 & 0.3449 & $0.079^{*}$ \\
\hline $\mathrm{C} 45$ & $1.1894(2)$ & $0.55561(10)$ & $0.7650(2)$ & $0.0428(5)$ \\
\hline $\mathrm{H} 45 \mathrm{~A}$ & 1.2151 & 0.5911 & 0.7556 & $0.064 *$ \\
\hline $\mathrm{H} 45 \mathrm{~B}$ & 1.2572 & 0.5316 & 0.7643 & $0.064 *$ \\
\hline $\mathrm{H} 45 \mathrm{C}$ & 1.1751 & 0.5526 & 0.8372 & $0.064 *$ \\
\hline $\mathrm{C} 46$ & $0.8585(2)$ & $0.72778(9)$ & $1.07566(17)$ & $0.0338(5)$ \\
\hline $\mathrm{C} 47$ & $0.7439(3)$ & $0.69620(10)$ & $1.0791(2)$ & $0.0415(6)$ \\
\hline H47A & 0.7534 & 0.6602 & 1.0588 & $0.062^{*}$ \\
\hline H47B & 0.6651 & 0.7107 & 1.0252 & $0.062^{*}$ \\
\hline $\mathrm{H} 47 \mathrm{C}$ & 0.7399 & 0.6975 & 1.1556 & $0.062^{*}$ \\
\hline $\mathrm{C} 48$ & $0.9848(3)$ & $0.70463(10)$ & $1.15472(19)$ & $0.0438(6)$ \\
\hline H48A & 0.9823 & 0.7021 & 1.2316 & $0.066^{*}$ \\
\hline H48B & 1.0560 & 0.7269 & 1.1552 & $0.066^{*}$ \\
\hline $\mathrm{H} 48 \mathrm{C}$ & 0.9965 & 0.6701 & 1.1279 & $0.066^{*}$ \\
\hline C49 & $0.8440(3)$ & $0.78176(9)$ & 1.11995 (19) & $0.0455(6)$ \\
\hline H49A & 0.8371 & 0.7787 & 1.1952 & $0.068^{*}$ \\
\hline H49B & 0.7667 & 0.7983 & 1.0681 & $0.068^{*}$ \\
\hline $\mathrm{H} 49 \mathrm{C}$ & 0.9188 & 0.8028 & 1.1249 & $0.068^{*}$ \\
\hline $\mathrm{C} 50$ & $0.8673(3)$ & $0.83944(9)$ & $0.6481(2)$ & $0.0445(6)$ \\
\hline H50A & 0.9270 & 0.8189 & 0.6237 & $0.067^{*}$ \\
\hline
\end{tabular}




\begin{tabular}{|c|c|c|c|c|}
\hline H50B & 0.8783 & 0.8762 & 0.6349 & $0.067^{*}$ \\
\hline $\mathrm{H} 50 \mathrm{C}$ & 0.7794 & 0.8290 & 0.6047 & $0.067^{*}$ \\
\hline C51 & $0.8631(2)$ & $0.51759(7)$ & $1.06368(16)$ & $0.0272(4)$ \\
\hline C52 & $0.9253(2)$ & $0.50443(7)$ & $0.96605(16)$ & $0.0258(4)$ \\
\hline $\mathrm{C} 53$ & $0.9628(2)$ & $0.52742(7)$ & $1.17970(17)$ & 0.0333 \\
\hline C54 & $0.9590(3)$ & $0.57220(9)$ & $1.23876(18)$ & $0.0436(6)$ \\
\hline H54 & 0.8948 & 0.5974 & 1.2056 & $0.052 *$ \\
\hline C55 & $1.0473(3)$ & $0.58061(11)$ & $1.3451(2)$ & $0.0635(8)$ \\
\hline H55 & 1.0434 & 0.6115 & 1.3845 & $0.076^{*}$ \\
\hline $\mathrm{C} 56$ & $1.1416(3)$ & $0.54451(14)$ & $1.3952(2)$ & $0.0684(9)$ \\
\hline H56 & 1.2029 & 0.5508 & 1.4681 & $0.082 *$ \\
\hline C57 & $1.1457(3)$ & $0.49955(12)$ & $1.3383(2)$ & $0.0553(7)$ \\
\hline H57 & 1.2091 & 0.4742 & 1.3722 & $0.066^{*}$ \\
\hline C58 & $1.0572(2)$ & $0.49143(9)$ & $1.23184(19)$ & $0.0410(5)$ \\
\hline $\mathrm{H} 58$ & 1.0609 & 0.4603 & 1.1930 & $0.049^{*}$ \\
\hline C59 & $0.7618(2)$ & $0.47876(8)$ & $1.06974(19)$ & $0.0297(5)$ \\
\hline $\mathrm{C} 60$ & $0.7693(2)$ & $0.45389(8)$ & $1.1700(2)$ & $0.0407(5)$ \\
\hline $\mathrm{H} 60$ & 0.8376 & 0.4619 & 1.2379 & $0.049 *$ \\
\hline C61 & $0.6786(2)$ & $0.41769(9)$ & $1.1717(2)$ & $0.0525(7)$ \\
\hline H61 & 0.6854 & 0.4009 & 1.2406 & $0.063 *$ \\
\hline C62 & $0.5784(3)$ & $0.40585(10)$ & $1.07396(18)$ & $0.0524(7)$ \\
\hline H62 & 0.5181 & 0.3800 & 1.0746 & $0.063 *$ \\
\hline C63 & $0.5666(2)$ & $0.43203(9)$ & $0.9752(2)$ & $0.0488(6)$ \\
\hline H63 & 0.4963 & 0.4250 & 0.9081 & $0.059 *$ \\
\hline C64 & $0.6570(2)$ & $0.46836(8)$ & $0.9739(2)$ & $0.0382(5)$ \\
\hline H64 & 0.6470 & 0.4866 & 0.9059 & $0.046^{*}$ \\
\hline C65 & $1.0696(2)$ & $0.51295(9)$ & $1.00494(17)$ & $0.0320(5)$ \\
\hline C66 & $1.1212(2)$ & $0.56104(10)$ & $1.04461(18)$ & $0.0391(5)$ \\
\hline H66 & 1.0658 & 0.5888 & 1.0457 & $0.047 *$ \\
\hline C67 & $1.25186(18)$ & $0.56925(11)$ & $1.0827(2)$ & $0.0546(7)$ \\
\hline H67 & 1.2854 & 0.6024 & 1.1102 & $0.065^{*}$ \\
\hline C68 & $1.3338(3)$ & $0.52943(10)$ & $1.0807(2)$ & $0.0651(9)$ \\
\hline H68 & 1.4237 & 0.5348 & 1.1078 & $0.078^{*}$ \\
\hline C69 & $1.2832(2)$ & $0.48177(11)$ & $1.0386(2)$ & $0.0635(9)$ \\
\hline H69 & 1.3387 & 0.4544 & 1.0355 & $0.076^{*}$ \\
\hline $\mathrm{C} 70$ & $1.1525(2)$ & $0.47359(10)$ & $1.0011(2)$ & $0.0458(6)$ \\
\hline $\mathrm{H} 70$ & 1.1191 & 0.4406 & 0.9724 & $0.055^{*}$ \\
\hline C71 & $0.8872(2)$ & $0.45146(8)$ & $0.91157(18)$ & $0.0300(4)$ \\
\hline $\mathrm{C} 72$ & $0.9014(2)$ & $0.40673(8)$ & $0.9771(2)$ & $0.0355(5)$ \\
\hline $\mathrm{H} 72$ & 0.9386 & 0.4089 & 1.0572 & $0.043^{*}$ \\
\hline $\mathrm{C} 73$ & $0.8617(3)$ & $0.35940(9)$ & $0.9266(2)$ & $0.0446(6)$ \\
\hline $\mathrm{H} 73$ & 0.8711 & 0.3293 & 0.9722 & $0.053^{*}$ \\
\hline C74 & 0.8087 (3) & $0.35539(10)$ & 0.8108 & $0.0591(8)$ \\
\hline $\mathrm{H} 74$ & 0.7785 & 0.3230 & 0.7761 & $0.071^{*}$ \\
\hline $\mathrm{C} 75$ & $0.7999(4)$ & $0.39903(10)$ & $0.7457(2)$ & $0.0620(8)$ \\
\hline $\mathrm{H} 75$ & 0.7664 & 0.3964 & 0.6655 & $0.074^{*}$ \\
\hline C76 & $0.8393(3)$ & $0.44637(9)$ & $0.79552(19)$ & $0.0419(6)$ \\
\hline H76 & 0.8334 & 0.4760 & 0.7492 & $0.050 *$ \\
\hline
\end{tabular}




$\begin{array}{lllll}\text { O1 } & 0.41561(13) & 0.67214(5) & 0.57154(11) & 0.0249(3) \\ \text { O2 } & 0.56444(13) & 0.72564(5) & 0.70404(11) & 0.0258(3) \\ \text { O3 } & 0.64584(13) & 0.66599(5) & 0.58506(11) & 0.0265(3) \\ \text { O4 } & 0.87143(14) & 0.63686(5) & 0.93978(11) & 0.0266(3) \\ \text { O5 } & 0.79305(14) & 0.56579(5) & 1.02459(11) & 0.0285(3) \\ \text { O6 } & 0.86838(14) & 0.54274(5) & 0.87786(11) & 0.0258(3) \\ \text { O7 } & 0.5764(3) & 0.49777(8) & 0.6399(2) & 0.0890(9) \\ \text { O8 } & 0.4804(2) & 0.60351(9) & 0.97680(18) & 0.0691(6) \\ \text { O9 } & 1.07528(16) & 0.54333(6) & 0.67518(14) & 0.0421(4) \\ \text { O10 } & 0.89204(16) & 0.83106(5) & 0.76520(13) & 0.0341(3) \\ \text { P1 } & 0.55672(5) & 0.66565(2) & 0.66426(4) & 0.02305(10) \\ \text { P2 } & 0.78003(5) & 0.586778(19) & 0.90123(4) & 0.02370(10) \\ \text { Rh1 } & 0.574142(14) & 0.594078(6) & 0.777762(12) & 0.02906(4) \\ \text { H1 } & 0.444(3) & 0.5921(15) & 0.708(2) & 0.062(7)^{*}\end{array}$

Atomic displacement parameters $\left(\AA^{2}\right)$

\begin{tabular}{|c|c|c|c|c|c|c|}
\hline & $U^{11}$ & $U^{22}$ & $U^{33}$ & $U^{12}$ & $U^{13}$ & $U^{23}$ \\
\hline $\mathrm{C} 1$ & $0.0549(18)$ & $0.0355(13)$ & $0.0473(15)$ & $-0.0018(12)$ & $-0.0045(13)$ & $0.0005(12)$ \\
\hline $\mathrm{C} 2$ & $0.0323(11)$ & $0.0520(15)$ & $0.0581(13)$ & $0.0060(12)$ & $0.0228(10)$ & $0.0182(13)$ \\
\hline $\mathrm{C} 3$ & $0.0249(10)$ & $0.0200(8)$ & $0.0234(9)$ & $0.0035(7)$ & $0.0074(7)$ & $0.0006(7)$ \\
\hline $\mathrm{C} 4$ & $0.0210(10)$ & $0.0247(9)$ & $0.0263(9)$ & $0.0047(7)$ & $0.0052(7)$ & $-0.0008(7)$ \\
\hline $\mathrm{C} 5$ & $0.0339(12)$ & $0.0267(9)$ & $0.0273(10)$ & $0.0069(8)$ & $0.0129(9)$ & $0.0036(8)$ \\
\hline C6 & $0.0387(13)$ & $0.0452(13)$ & $0.0293(11)$ & $0.0082(10)$ & $0.0117(9)$ & $0.0003(9)$ \\
\hline $\mathrm{C} 7$ & $0.0610(18)$ & $0.0641(17)$ & $0.0280(11)$ & $0.0204(14)$ & $0.0166(11)$ & $0.0042(11)$ \\
\hline $\mathrm{C} 8$ & $0.076(2)$ & $0.0670(17)$ & 0.0435 (14) & $0.0223(16)$ & $0.0392(15)$ & $0.0192(13)$ \\
\hline C9 & $0.0569(17)$ & 0.0479 (14) & $0.0683(18)$ & $0.0032(12)$ & $0.0427(15)$ & $0.0115(12)$ \\
\hline $\mathrm{C} 10$ & $0.0402(13)$ & $0.0351(11)$ & $0.0430(12)$ & $0.0011(10)$ & $0.0215(11)$ & $0.0007(9)$ \\
\hline C11 & $0.0260(10)$ & $0.0246(9)$ & $0.0206(8)$ & $0.0017(8)$ & $0.0063(7)$ & $-0.0038(7)$ \\
\hline $\mathrm{C} 12$ & $0.0292(11)$ & $0.0289(10)$ & $0.0298(10)$ & $0.0006(8)$ & $0.0086(8)$ & $-0.0012(8)$ \\
\hline $\mathrm{C} 13$ & $0.0319(12)$ & $0.0392(11)$ & $0.0353(11)$ & $-0.0044(9)$ & $0.0148(9)$ & $-0.0018(9)$ \\
\hline $\mathrm{C} 14$ & $0.0250(11)$ & $0.0433(12)$ & $0.0345(11)$ & $0.0029(9)$ & $0.0084(9)$ & $-0.0090(9)$ \\
\hline $\mathrm{C} 15$ & $0.0284(11)$ & $0.0361(11)$ & $0.0297(10)$ & $0.0103(9)$ & $0.0010(8)$ & $-0.0031(8)$ \\
\hline $\mathrm{C} 16$ & $0.0299(11)$ & $0.0272(10)$ & $0.0268(10)$ & $0.0024(8)$ & $0.0062(8)$ & $0.0006(7)$ \\
\hline $\mathrm{C} 17$ & $0.0279(11)$ & $0.0267(9)$ & $0.0242(9)$ & $0.0002(8)$ & $0.0069(8)$ & $-0.0060(7)$ \\
\hline $\mathrm{C} 18$ & $0.0301(11)$ & $0.0311(10)$ & $0.0324(11)$ & $0.0047(9)$ & $0.0092(9)$ & $-0.0064(8)$ \\
\hline C19 & $0.0338(13)$ & $0.0451(13)$ & $0.0468(13)$ & $0.0075(10)$ & $0.0189(10)$ & $-0.0047(10)$ \\
\hline $\mathrm{C} 20$ & 0.0677 (19) & $0.0566(16)$ & $0.0576(16)$ & $0.0150(14)$ & $0.0463(15)$ & $0.0055(13)$ \\
\hline $\mathrm{C} 21$ & $0.093(2)$ & $0.0676(18)$ & $0.0553(17)$ & $0.0359(18)$ & $0.0504(17)$ & $0.0264(14)$ \\
\hline $\mathrm{C} 22$ & $0.0567(16)$ & $0.0516(14)$ & $0.0386(12)$ & $0.0277(12)$ & $0.0241(12)$ & $0.0081(10)$ \\
\hline $\mathrm{C} 23$ & $0.0251(11)$ & $0.0271(9)$ & $0.0362(11)$ & $-0.0006(8)$ & $0.0136(9)$ & $-0.0079(8)$ \\
\hline $\mathrm{C} 24$ & $0.0422(14)$ & $0.0289(10)$ & $0.0471(13)$ & $-0.0032(10)$ & $0.0106(11)$ & $0.0014(9)$ \\
\hline $\mathrm{C} 25$ & $0.0583(18)$ & $0.0297(11)$ & $0.0613(16)$ & $-0.0021(11)$ & $0.0217(14)$ & $0.0027(11)$ \\
\hline $\mathrm{C} 26$ & $0.0515(17)$ & $0.0288(11)$ & 0.0818 (19) & $-0.0114(11)$ & $0.0359(15)$ & $-0.0152(12)$ \\
\hline $\mathrm{C} 27$ & $0.0357(14)$ & $0.0423(13)$ & $0.0729(18)$ & $-0.0080(11)$ & $0.0179(13)$ & $-0.0277(13)$ \\
\hline $\mathrm{C} 28$ & $0.0309(12)$ & $0.0329(11)$ & 0.0449 (12) & $0.0017(9)$ & $0.0076(10)$ & $-0.0102(9)$ \\
\hline $\mathrm{C} 29$ & $0.0230(10)$ & $0.0228(8)$ & $0.0267(9)$ & $0.0005(7)$ & $0.0108(8)$ & $0.0015(7)$ \\
\hline $\mathrm{C} 30$ & $0.0261(8)$ & $0.0258(8)$ & $0.0289(8)$ & $0.0002(11)$ & $0.0098(7)$ & $-0.0012(10)$ \\
\hline
\end{tabular}




\begin{tabular}{|c|c|c|c|c|c|c|}
\hline C31 & $0.0312(11)$ & $0.0252(10)$ & $0.0340(10)$ & $0.0036(8)$ & $0.0126(9)$ & $-0.0061(8)$ \\
\hline C32 & $0.0262(11)$ & $0.0260(9)$ & $0.0347(10)$ & $0.0041(8)$ & $0.0135(8)$ & $0.0000(8)$ \\
\hline C33 & $0.0219(10)$ & $0.0274(8)$ & $0.0265(9)$ & $-0.0022(7)$ & $0.0083(8)$ & $0.0014(7)$ \\
\hline C34 & $0.0249(10)$ & $0.0205(8)$ & $0.0254(9)$ & $-0.0012(7)$ & $0.0127(8)$ & $0.0005(7)$ \\
\hline C35 & $0.0199(10)$ & $0.0258(9)$ & $0.0259(9)$ & $-0.0003(7)$ & $0.0070(7)$ & $-0.0029(7)$ \\
\hline C36 & $0.0223(10)$ & $0.0243(9)$ & $0.0259(9)$ & $-0.0024(7)$ & $0.0089(8)$ & $0.0004(7)$ \\
\hline C37 & $0.0220(10)$ & $0.0302(10)$ & $0.0233(9)$ & $-0.0022(8)$ & $0.0045(8)$ & $-0.0037(8)$ \\
\hline C38 & $0.0255(11)$ & $0.0278(10)$ & $0.0298(10)$ & $-0.0024(8)$ & $0.0066(8)$ & $-0.0080(8)$ \\
\hline C39 & $0.0271(11)$ & $0.0231(9)$ & $0.0341(10)$ & -0.0015 & $0.0123(9)$ & $0.0018(8)$ \\
\hline $\mathrm{C} 40$ & $0.0256(11)$ & $0.0255(9)$ & $0.0266(9)$ & $-0.0002(8)$ & $0.0101(8)$ & $0.0005(7)$ \\
\hline $\mathrm{C} 41$ & $0.0315(10)$ & 0.0393 (12) & $0.0298(9)$ & $0.0041(11)$ & $0.0061(7)$ & $-0.0084(10)$ \\
\hline $\mathrm{C} 42$ & $0.069(2)$ & 0.0575 (17) & $0.0351(14)$ & $-0.0011(15)$ & $0.0024(13)$ & $-0.0005(12)$ \\
\hline $\mathrm{C} 43$ & $0.0320(12)$ & $0.081(2)$ & $0.0413(12)$ & $-0.0052(13)$ & $0.0050(9)$ & $-0.0233(13)$ \\
\hline $\mathrm{C} 44$ & $0.0440(17)$ & $0.0582(17)$ & $0.0421(14)$ & $0.0096(13)$ & $-0.0032(12)$ & $-0.0272(12)$ \\
\hline $\mathrm{C} 45$ & $0.0266(12)$ & 0.0497 (13) & $0.0498(14)$ & $0.0097(10)$ & $0.0102(10)$ & $0.0030(11)$ \\
\hline $\mathrm{C} 46$ & 0.0405 & $0.0381(11)$ & $0.0235(9)$ & $-0.0011(9)$ & $0.0117(9)$ & $-0.0047(8)$ \\
\hline $\mathrm{C} 47$ & $0.0504(16)$ & $0.0441(14)$ & $0.0369(13)$ & $-0.0003(12)$ & $0.0238(12)$ & $-0.0023(10)$ \\
\hline $\mathrm{C} 48$ & $0.0474(15)$ & $0.0489(14)$ & $0.0285(11)$ & $0.0017(11)$ & $0.0049(10)$ & $-0.0038(10)$ \\
\hline C49 & $0.0662(18)$ & $0.0410(12)$ & $0.0310(11)$ & $0.0029(12)$ & $0.0191(11)$ & $-0.0073(9)$ \\
\hline C50 & $0.0620(17)$ & $0.0319(11)$ & $0.0430(13)$ & $-0.0021(11)$ & $0.0225(12)$ & $0.0072(9)$ \\
\hline C51 & $0.0318(11)$ & $0.0258(9)$ & $0.0262(9)$ & $0.0054(8)$ & $0.0128(8)$ & $0.0046(7)$ \\
\hline C52 & $0.0262(11)$ & $0.0271(9)$ & $0.0250(9)$ & $0.0050(8)$ & $0.0103(8)$ & $0.0060(7)$ \\
\hline C53 & $0.0379(13)$ & 0.0385 (11) & $0.0250(10)$ & $0.0000(9)$ & $0.0127(9)$ & $0.0043(8)$ \\
\hline C54 & $0.0526(16)$ & $0.0460(12)$ & $0.0314(11)$ & $0.0057(11)$ & $0.0136(10)$ & $-0.0014(9)$ \\
\hline C55 & $0.084(2)$ & $0.062(2)$ & $0.0370(12)$ & $0.0020(15)$ & 0.0107 (13) & $-0.0136(12)$ \\
\hline C56 & $0.072(2)$ & $0.089(2)$ & 0.0295 (13) & $0.0022(18)$ & $-0.0022(13)$ & $-0.0054(14)$ \\
\hline C57 & $0.0528(17)$ & $0.0710(19)$ & $0.0356(13)$ & $0.0133(14)$ & $0.0069(12)$ & $0.0103(12)$ \\
\hline C58 & $0.0445(14)$ & $0.0481(13)$ & $0.0293(11)$ & $0.0094(11)$ & $0.0112(10)$ & $0.0067(10)$ \\
\hline C59 & $0.0369(13)$ & $0.0248(10)$ & $0.0343(11)$ & $0.0063(9)$ & $0.0211(10)$ & $0.0057(8)$ \\
\hline C60 & $0.0554(16)$ & $0.0352(11)$ & 0.0409 (12) & $0.0044(11)$ & $0.0287(12)$ & $0.0091(10)$ \\
\hline C61 & $0.071(2)$ & 0.0415 (13) & $0.0588(16)$ & $0.0022(13)$ & 0.0407 (16) & $0.0163(12)$ \\
\hline C62 & $0.0511(17)$ & $0.0388(13)$ & $0.081(2)$ & $-0.0036(12)$ & $0.0399(16)$ & $0.0117(13)$ \\
\hline C63 & $0.0361(14)$ & 0.0475 (14) & 0.0645 (17) & $-0.0049(11)$ & $0.0196(13)$ & $0.0049(12)$ \\
\hline C64 & $0.0335(13)$ & $0.0384(12)$ & $0.0461(13)$ & $0.0018(10)$ & $0.0180(11)$ & $0.0111(10)$ \\
\hline C65 & $0.0288(11)$ & $0.0412(12)$ & $0.0262(10)$ & $0.0039(9)$ & $0.0098(8)$ & $0.0104(8)$ \\
\hline C66 & $0.0323(12)$ & $0.0510(13)$ & $0.0320(11)$ & $-0.0076(10)$ & $0.0084(9)$ & $0.0029(10)$ \\
\hline C67 & $0.0355(14)$ & $0.0747(18)$ & $0.0444(14)$ & $-0.0131(13)$ & $0.0023(11)$ & $0.0148(13)$ \\
\hline C68 & $0.0260(14)$ & $0.100(2)$ & $0.0613(17)$ & $-0.0023(15)$ & $0.0052(12)$ & $0.0395(17)$ \\
\hline C69 & $0.0347(15)$ & $0.081(2)$ & $0.081(2)$ & $0.0246(15)$ & $0.0285(15)$ & $0.0445(17)$ \\
\hline C70 & $0.0333(13)$ & $0.0500(14)$ & $0.0572(15)$ & $0.0129(11)$ & $0.0196(11)$ & $0.0210(12)$ \\
\hline C71 & $0.0294(12)$ & $0.0295(10)$ & $0.0356(11)$ & $0.0037(8)$ & $0.0170(9)$ & $0.0009(8)$ \\
\hline $\mathrm{C} 72$ & 0.0394 (13) & 0.0304 (11) & $0.0401(12)$ & $0.0096(9)$ & $0.0180(10)$ & $0.0074(9)$ \\
\hline C73 & $0.0563(16)$ & $0.0260(10)$ & $0.0588(15)$ & $0.0044(10)$ & $0.0294(13)$ & $0.0051(10)$ \\
\hline $\mathrm{C} 74$ & $0.083(2)$ & $0.0314(12)$ & $0.0650(18)$ & $-0.0071(13)$ & $0.0281(17)$ & $-0.0115(12)$ \\
\hline $\mathrm{C} 75$ & $0.096(3)$ & $0.0431(14)$ & $0.0418(14)$ & $-0.0058(15)$ & $0.0176(16)$ & $-0.0098(11)$ \\
\hline C76 & $0.0628(17)$ & $0.0309(11)$ & $0.0324(11)$ & $0.0014(11)$ & $0.0169(11)$ & $0.0003(9)$ \\
\hline $\mathrm{O} 1$ & $0.0233(7)$ & $0.0216(6)$ & $0.0277(7)$ & $0.0013(5)$ & $0.0060(5)$ & $-0.0010(5)$ \\
\hline $\mathrm{O} 2$ & $0.0208(7)$ & $0.0273(7)$ & $0.0261(6)$ & $0.0041(5)$ & $0.0041(5)$ & $-0.0031(5)$ \\
\hline
\end{tabular}




\begin{tabular}{lllllll} 
O3 & $0.0251(7)$ & $0.0269(6)$ & $0.0270(7)$ & $0.0056(6)$ & $0.0084(6)$ & $-0.0006(5)$ \\
O4 & $0.0296(8)$ & $0.0247(7)$ & $0.0222(7)$ & $-0.0006(6)$ & $0.0047(6)$ & $0.0021(5)$ \\
O5 & $0.0336(8)$ & $0.0256(7)$ & $0.0291(7)$ & $0.0061(6)$ & $0.0145(6)$ & $0.0048(5)$ \\
O6 & $0.0300(8)$ & $0.0251(7)$ & $0.0225(6)$ & $0.0036(6)$ & $0.0092(6)$ & $0.0038(5)$ \\
O7 & $0.115(2)$ & $0.0415(11)$ & $0.0759(15)$ & $0.0036(13)$ & $-0.0104(14)$ & $-0.0194(11)$ \\
O8 & $0.0672(13)$ & $0.0842(17)$ & $0.0769(13)$ & $0.0133(12)$ & $0.0514(11)$ & $0.0264(12)$ \\
O9 & $0.0313(9)$ & $0.0427(9)$ & $0.0463(9)$ & $0.0127(7)$ & $0.0056(7)$ & $-0.0105(7)$ \\
O10 & $0.0423(9)$ & $0.0219(7)$ & $0.0406(8)$ & $-0.0021(6)$ & $0.0176(7)$ & $0.0014(6)$ \\
P1 & $0.0218(3)$ & $0.0234(2)$ & $0.0234(2)$ & $0.0023(2)$ & $0.0071(2)$ & $0.00024(19)$ \\
P2 & $0.0243(2)$ & $0.0229(3)$ & $0.0240(2)$ & $0.0010(2)$ & $0.00854(17)$ & $0.00250(18)$ \\
Rh1 & $0.02251(7)$ & $0.02832(7)$ & $0.03357(7)$ & $-0.00073(8)$ & $0.00617(5)$ & $0.00755(8)$ \\
\hline
\end{tabular}

Geometric parameters $\left(\AA,{ }^{\circ}\right)$

\begin{tabular}{|c|c|c|c|}
\hline $\mathrm{C} 77-\mathrm{C} 78$ & $1.414(5)$ & $\mathrm{C} 41-\mathrm{C} 42$ & $1.520(4)$ \\
\hline C77-H77A & 0.9800 & $\mathrm{C} 41-\mathrm{C} 43$ & $1.523(3)$ \\
\hline C77-H77B & 0.9800 & $\mathrm{C} 41-\mathrm{C} 44$ & $1.537(3)$ \\
\hline $\mathrm{C} 77-\mathrm{H} 77 \mathrm{C}$ & 0.9800 & $\mathrm{C} 42-\mathrm{H} 42 \mathrm{~A}$ & 0.9800 \\
\hline $\mathrm{C} 78-\mathrm{O} 11$ & $1.369(4)$ & $\mathrm{C} 42-\mathrm{H} 42 \mathrm{~B}$ & 0.9800 \\
\hline $\mathrm{C} 78-\mathrm{H} 78 \mathrm{~A}$ & 0.9900 & $\mathrm{C} 42-\mathrm{H} 42 \mathrm{C}$ & 0.9800 \\
\hline $\mathrm{C} 78-\mathrm{H} 78 \mathrm{~B}$ & 0.9900 & $\mathrm{C} 43-\mathrm{H} 43 \mathrm{~A}$ & 0.9800 \\
\hline $\mathrm{C} 79-\mathrm{O} 11$ & $1.377(4)$ & $\mathrm{C} 43-\mathrm{H} 43 \mathrm{~B}$ & 0.9800 \\
\hline $\mathrm{C} 79-\mathrm{C} 80$ & $1.415(5)$ & $\mathrm{C} 43-\mathrm{H} 43 \mathrm{C}$ & 0.9800 \\
\hline C79-H79A & 0.9900 & $\mathrm{C} 44-\mathrm{H} 44 \mathrm{~A}$ & 0.9800 \\
\hline C79-H79B & 0.9900 & $\mathrm{C} 44-\mathrm{H} 44 \mathrm{~B}$ & 0.9800 \\
\hline $\mathrm{C} 80-\mathrm{H} 80 \mathrm{~A}$ & 0.9800 & $\mathrm{C} 44-\mathrm{H} 44 \mathrm{C}$ & 0.9800 \\
\hline $\mathrm{C} 80-\mathrm{H} 80 \mathrm{~B}$ & 0.9800 & $\mathrm{C} 45-\mathrm{O} 9$ & $1.415(3)$ \\
\hline $\mathrm{C} 80-\mathrm{H} 80 \mathrm{C}$ & 0.9800 & $\mathrm{C} 45-\mathrm{H} 45 \mathrm{~A}$ & 0.9800 \\
\hline $\mathrm{C} 1-\mathrm{O} 7$ & $1.132(3)$ & $\mathrm{C} 45-\mathrm{H} 45 \mathrm{~B}$ & 0.9800 \\
\hline $\mathrm{C} 1-\mathrm{Rh} 1$ & $1.909(3)$ & $\mathrm{C} 45-\mathrm{H} 45 \mathrm{C}$ & 0.9800 \\
\hline $\mathrm{C} 2-\mathrm{O} 8$ & $1.133(3)$ & $\mathrm{C} 46-\mathrm{C} 47$ & $1.529(4)$ \\
\hline $\mathrm{C} 2-\mathrm{Rh} 1$ & $1.909(2)$ & $\mathrm{C} 46-\mathrm{C} 49$ & $1.532(3)$ \\
\hline $\mathrm{C} 3-\mathrm{O} 1$ & $1.464(2)$ & $\mathrm{C} 46-\mathrm{C} 48$ & $1.536(3)$ \\
\hline $\mathrm{C} 3-\mathrm{C} 11$ & $1.528(3)$ & $\mathrm{C} 47-\mathrm{H} 47 \mathrm{~A}$ & 0.9800 \\
\hline $\mathrm{C} 3-\mathrm{C} 5$ & $1.531(3)$ & $\mathrm{C} 47-\mathrm{H} 47 \mathrm{~B}$ & 0.9800 \\
\hline $\mathrm{C} 3-\mathrm{C} 4$ & $1.604(3)$ & $\mathrm{C} 47-\mathrm{H} 47 \mathrm{C}$ & 0.9800 \\
\hline $\mathrm{C} 4-\mathrm{O} 2$ & $1.453(2)$ & $\mathrm{C} 48-\mathrm{H} 48 \mathrm{~A}$ & 0.9800 \\
\hline $\mathrm{C} 4-\mathrm{C} 23$ & $1.524(3)$ & $\mathrm{C} 48-\mathrm{H} 48 \mathrm{~B}$ & 0.9800 \\
\hline $\mathrm{C} 4-\mathrm{C} 17$ & $1.537(3)$ & $\mathrm{C} 48-\mathrm{H} 48 \mathrm{C}$ & 0.9800 \\
\hline $\mathrm{C} 5-\mathrm{C} 6$ & $1.388(3)$ & $\mathrm{C} 49-\mathrm{H} 49 \mathrm{~A}$ & 0.9800 \\
\hline $\mathrm{C} 5-\mathrm{C} 10$ & $1.398(3)$ & С49- Н49B & 0.9800 \\
\hline $\mathrm{C} 6-\mathrm{C} 7$ & $1.3814(18)$ & $\mathrm{C} 49-\mathrm{H} 49 \mathrm{C}$ & 0.9800 \\
\hline C6- 6 6 & 0.9500 & $\mathrm{C} 50-\mathrm{O} 10$ & $1.421(3)$ \\
\hline $\mathrm{C} 7-\mathrm{C} 8$ & 1.3797 (19) & $\mathrm{C} 50-\mathrm{H} 50 \mathrm{~A}$ & 0.9800 \\
\hline $\mathrm{C} 7-\mathrm{H} 7$ & 0.9500 & $\mathrm{C} 50-\mathrm{H} 50 \mathrm{~B}$ & 0.9800 \\
\hline $\mathrm{C} 8-\mathrm{C} 9$ & $1.3811(19)$ & $\mathrm{C} 50-\mathrm{H} 50 \mathrm{C}$ & 0.9800 \\
\hline $\mathrm{C} 8-\mathrm{H} 8$ & 0.9500 & $\mathrm{C} 51-\mathrm{O} 5$ & $1.462(2)$ \\
\hline $\mathrm{C} 9-\mathrm{C} 10$ & $1.3811(18)$ & $\mathrm{C} 51-\mathrm{C} 53$ & $1.520(3)$ \\
\hline
\end{tabular}




\begin{tabular}{|c|c|c|c|}
\hline $\mathrm{C} 9-\mathrm{H} 9$ & 0.9500 & $\mathrm{C} 51-\mathrm{C} 59$ & $1.532(3)$ \\
\hline $\mathrm{C} 10-\mathrm{H} 10$ & 0.9500 & $\mathrm{C} 51-\mathrm{C} 52$ & $1.643(3)$ \\
\hline $\mathrm{C} 11-\mathrm{C} 12$ & $1.387(3)$ & $\mathrm{C} 52-\mathrm{O} 6$ & $1.459(2)$ \\
\hline $\mathrm{C} 11-\mathrm{C} 16$ & $1.392(3)$ & $\mathrm{C} 52-\mathrm{C} 71$ & $1.524(3)$ \\
\hline $\mathrm{C} 12-\mathrm{C} 13$ & $1.390(3)$ & $\mathrm{C} 52-\mathrm{C} 65$ & $1.527(3)$ \\
\hline $\mathrm{C} 12-\mathrm{H} 12$ & 0.9500 & $\mathrm{C} 53-\mathrm{C} 54$ & $1.385(2)$ \\
\hline $\mathrm{C} 13-\mathrm{C} 14$ & $1.378(3)$ & $\mathrm{C} 53-\mathrm{C} 58$ & 1.387 (2) \\
\hline $\mathrm{C} 13-\mathrm{H} 13$ & 0.9500 & $\mathrm{C} 54-\mathrm{C} 55$ & $1.377(4)$ \\
\hline $\mathrm{C} 14-\mathrm{C} 15$ & $1.372(3)$ & $\mathrm{C} 54-\mathrm{H} 54$ & 0.9500 \\
\hline $\mathrm{C} 14-\mathrm{H} 14$ & 0.9500 & $\mathrm{C} 55-\mathrm{C} 56$ & $1.384(4)$ \\
\hline $\mathrm{C} 15-\mathrm{C} 16$ & $1.382(3)$ & C55-H55 & 0.9500 \\
\hline $\mathrm{C} 15-\mathrm{H} 15$ & 0.9500 & $\mathrm{C} 56-\mathrm{C} 57$ & $1.374(4)$ \\
\hline $\mathrm{C} 16-\mathrm{H} 16$ & 0.9500 & $\mathrm{C} 56-\mathrm{H} 56$ & 0.9500 \\
\hline $\mathrm{C} 17-\mathrm{C} 22$ & $1.386(2)$ & C57-C58 & $1.378(4)$ \\
\hline $\mathrm{C} 17-\mathrm{C} 18$ & $1.391(2)$ & C57-H57 & 0.9500 \\
\hline $\mathrm{C} 18-\mathrm{C} 19$ & $1.3779(18)$ & C58-H58 & 0.9500 \\
\hline $\mathrm{C} 18-\mathrm{H} 18$ & 0.9500 & C59-C64 & 1.385 \\
\hline $\mathrm{C} 19-\mathrm{C} 20$ & $1.3754(18)$ & $\mathrm{C} 59-\mathrm{C} 60$ & $1.393(3)$ \\
\hline $\mathrm{C} 19-\mathrm{H} 19$ & 0.9500 & $\mathrm{C} 60-\mathrm{C} 61$ & $1.3835(19)$ \\
\hline $\mathrm{C} 20-\mathrm{C} 21$ & $1.3751(18)$ & $\mathrm{C} 60-\mathrm{H} 60$ & 0.9500 \\
\hline $\mathrm{C} 20-\mathrm{H} 20$ & 0.9500 & C61-C62 & $1.3805(19)$ \\
\hline $\mathrm{C} 21-\mathrm{C} 22$ & $1.3778(18)$ & C61-H61 & 0.9500 \\
\hline $\mathrm{C} 21-\mathrm{H} 21$ & 0.9500 & C62-C63 & $1.3817(19)$ \\
\hline $\mathrm{C} 22-\mathrm{H} 22$ & 0.9500 & $\mathrm{C} 62-\mathrm{H} 62$ & 0.9500 \\
\hline $\mathrm{C} 23-\mathrm{C} 28$ & $1.385(3)$ & C63-C64 & $1.3814(19)$ \\
\hline $\mathrm{C} 23-\mathrm{C} 24$ & $1.389(3)$ & C63-H63 & 0.9500 \\
\hline $\mathrm{C} 24-\mathrm{C} 25$ & $1.3800(18)$ & C64-H64 & 0.9500 \\
\hline $\mathrm{C} 24-\mathrm{H} 24$ & 0.9500 & $\mathrm{C} 65-\mathrm{C} 70$ & $1.386(3)$ \\
\hline $\mathrm{C} 25-\mathrm{C} 26$ & $1.3791(18)$ & $\mathrm{C} 65-\mathrm{C} 66$ & $1.388(3)$ \\
\hline $\mathrm{C} 25-\mathrm{H} 25$ & 0.9500 & $\mathrm{C} 66-\mathrm{C} 67$ & $1.3835(19)$ \\
\hline $\mathrm{C} 26-\mathrm{C} 27$ & $1.3775(18)$ & C66-H66 & 0.9500 \\
\hline $\mathrm{C} 26-\mathrm{H} 26$ & 0.9500 & C67-C68 & $1.3823(19)$ \\
\hline $\mathrm{C} 27-\mathrm{C} 28$ & $1.3802(18)$ & C67-H67 & 0.9500 \\
\hline $\mathrm{C} 27-\mathrm{H} 27$ & 0.9500 & $\mathrm{C} 68-\mathrm{C} 69$ & $1.382(2)$ \\
\hline $\mathrm{C} 28-\mathrm{H} 28$ & 0.9500 & C68-H68 & 0.9500 \\
\hline $\mathrm{C} 29-\mathrm{O} 3$ & $1.396(2)$ & $\mathrm{C} 69-\mathrm{C} 70$ & $1.3840(19)$ \\
\hline $\mathrm{C} 29-\mathrm{C} 34$ & $1.397(3)$ & C69-H69 & 0.9500 \\
\hline $\mathrm{C} 29-\mathrm{C} 30$ & $1.400(3)$ & $\mathrm{C} 70-\mathrm{H} 70$ & 0.9500 \\
\hline $\mathrm{C} 30-\mathrm{C} 31$ & $1.398(3)$ & C71-C76 & $1.378(3)$ \\
\hline $\mathrm{C} 30-\mathrm{C} 41$ & $1.545(2)$ & $\mathrm{C} 71-\mathrm{C} 72$ & 1.397 (3) \\
\hline $\mathrm{C} 31-\mathrm{C} 32$ & $1.391(3)$ & $\mathrm{C} 72-\mathrm{C} 73$ & $1.379(3)$ \\
\hline $\mathrm{C} 31-\mathrm{H} 31$ & 0.9500 & $\mathrm{C} 72-\mathrm{H} 72$ & 0.9500 \\
\hline $\mathrm{C} 32-\mathrm{O} 9$ & $1.358(2)$ & $\mathrm{C} 73-\mathrm{C} 74$ & $1.375(4)$ \\
\hline $\mathrm{C} 32-\mathrm{C} 33$ & $1.382(3)$ & $\mathrm{C} 73-\mathrm{H} 73$ & 0.9500 \\
\hline $\mathrm{C} 33-\mathrm{C} 34$ & $1.393(3)$ & $\mathrm{C} 74-\mathrm{C} 75$ & $1.378(4)$ \\
\hline C $33-\mathrm{H} 33$ & 0.9500 & $\mathrm{C} 74-\mathrm{H} 74$ & 0.9500 \\
\hline $\mathrm{C} 34-\mathrm{C} 35$ & $1.485(3)$ & $\mathrm{C} 75-\mathrm{C} 76$ & $1.376(3)$ \\
\hline $\mathrm{C} 35-\mathrm{C} 36$ & $1.383(3)$ & $\mathrm{C} 75-\mathrm{H} 75$ & 0.9500 \\
\hline
\end{tabular}




\begin{tabular}{|c|c|c|c|}
\hline $\mathrm{C} 35-\mathrm{C} 40$ & $1.402(3)$ & $\mathrm{C} 76-\mathrm{H} 76$ & 0.9500 \\
\hline $\mathrm{C} 36-\mathrm{O} 4$ & $1.403(2)$ & $\mathrm{O} 1-\mathrm{P} 1$ & $1.6106(14)$ \\
\hline $\mathrm{C} 36-\mathrm{C} 37$ & $1.407(3)$ & $\mathrm{O} 2-\mathrm{P} 1$ & $1.6224(14)$ \\
\hline $\mathrm{C} 37-\mathrm{C} 38$ & $1.375(3)$ & $\mathrm{O} 3-\mathrm{P} 1$ & $1.6336(14)$ \\
\hline $\mathrm{C} 37-\mathrm{C} 46$ & $1.548(3)$ & $\mathrm{O} 4-\mathrm{P} 2$ & $1.6146(14)$ \\
\hline C38-C39 & $1.394(3)$ & $\mathrm{O} 5-\mathrm{P} 2$ & $1.6039(14)$ \\
\hline $\mathrm{C} 38-\mathrm{H} 38$ & 0.9500 & $\mathrm{O} 6-\mathrm{P} 2$ & $1.5979(14)$ \\
\hline $\mathrm{C} 39-\mathrm{O} 10$ & $1.367(2)$ & $\mathrm{P} 1-\mathrm{Rh} 1$ & $2.3045(5)$ \\
\hline $\mathrm{C} 39-\mathrm{C} 40$ & $1.375(3)$ & $\mathrm{P} 2-\mathrm{Rh} 1$ & $2.2913(5)$ \\
\hline $\mathrm{C} 40-\mathrm{H} 40$ & 0.9500 & $\mathrm{Rh} 1-\mathrm{H} 1$ & $1.42(3)$ \\
\hline $\mathrm{C} 78-\mathrm{C} 77-\mathrm{H} 77 \mathrm{~A}$ & 109.5 & $\mathrm{H} 43 \mathrm{~A}-\mathrm{C} 43-\mathrm{H} 43 \mathrm{~B}$ & 109.5 \\
\hline C78-C77-H77B & 109.5 & $\mathrm{C} 41-\mathrm{C} 43-\mathrm{H} 43 \mathrm{C}$ & 109.5 \\
\hline H77A-C77-H77B & 109.5 & $\mathrm{H} 43 \mathrm{~A}-\mathrm{C} 43-\mathrm{H} 43 \mathrm{C}$ & 109.5 \\
\hline C78-C77-H77C & 109.5 & $\mathrm{H} 43 \mathrm{~B}-\mathrm{C} 43-\mathrm{H} 43 \mathrm{C}$ & 109.5 \\
\hline $\mathrm{H} 77 \mathrm{~A}-\mathrm{C} 77-\mathrm{H} 77 \mathrm{C}$ & 109.5 & $\mathrm{C} 41-\mathrm{C} 44-\mathrm{H} 44 \mathrm{~A}$ & 109.5 \\
\hline $\mathrm{H} 77 \mathrm{~B}-\mathrm{C} 77-\mathrm{H} 77 \mathrm{C}$ & 109.5 & $\mathrm{C} 41-\mathrm{C} 44-\mathrm{H} 44 \mathrm{~B}$ & 109.5 \\
\hline $\mathrm{O} 11-\mathrm{C} 78-\mathrm{C} 77$ & $116.2(4)$ & $\mathrm{H} 44 \mathrm{~A}-\mathrm{C} 44-\mathrm{H} 44 \mathrm{~B}$ & 109.5 \\
\hline $\mathrm{O} 11-\mathrm{C} 78-\mathrm{H} 78 \mathrm{~A}$ & 108.2 & $\mathrm{C} 41-\mathrm{C} 44-\mathrm{H} 44 \mathrm{C}$ & 109.5 \\
\hline $\mathrm{C} 77-\mathrm{C} 78-\mathrm{H} 78 \mathrm{~A}$ & 108.2 & $\mathrm{H} 44 \mathrm{~A}-\mathrm{C} 44-\mathrm{H} 44 \mathrm{C}$ & 109.5 \\
\hline $\mathrm{O} 11-\mathrm{C} 78-\mathrm{H} 78 \mathrm{~B}$ & 108.2 & $\mathrm{H} 44 \mathrm{~B}-\mathrm{C} 44-\mathrm{H} 44 \mathrm{C}$ & 109.5 \\
\hline $\mathrm{C} 77-\mathrm{C} 78-\mathrm{H} 78 \mathrm{~B}$ & 108.2 & $\mathrm{O} 9-\mathrm{C} 45-\mathrm{H} 45 \mathrm{~A}$ & 109.5 \\
\hline $\mathrm{H} 78 \mathrm{~A}-\mathrm{C} 78-\mathrm{H} 78 \mathrm{~B}$ & 107.4 & $\mathrm{O} 9-\mathrm{C} 45-\mathrm{H} 45 \mathrm{~B}$ & 109.5 \\
\hline $\mathrm{O} 11-\mathrm{C} 79-\mathrm{C} 80$ & $109.3(5)$ & $\mathrm{H} 45 \mathrm{~A}-\mathrm{C} 45-\mathrm{H} 45 \mathrm{~B}$ & 109.5 \\
\hline O11-C79-H79A & 109.8 & $\mathrm{O} 9-\mathrm{C} 45-\mathrm{H} 45 \mathrm{C}$ & 109.5 \\
\hline $\mathrm{C} 80-\mathrm{C} 79-\mathrm{H} 79 \mathrm{~A}$ & 109.8 & $\mathrm{H} 45 \mathrm{~A}-\mathrm{C} 45-\mathrm{H} 45 \mathrm{C}$ & 109.5 \\
\hline О11-С79-Н79B & 109.8 & $\mathrm{H} 45 \mathrm{~B}-\mathrm{C} 45-\mathrm{H} 45 \mathrm{C}$ & 109.5 \\
\hline С $80-\mathrm{C} 79-\mathrm{H} 79 \mathrm{~B}$ & 109.8 & $\mathrm{C} 47-\mathrm{C} 46-\mathrm{C} 49$ & $106.3(2)$ \\
\hline H79A-C79-H79B & 108.3 & $\mathrm{C} 47-\mathrm{C} 46-\mathrm{C} 48$ & $111.1(2)$ \\
\hline $\mathrm{C} 79-\mathrm{C} 80-\mathrm{H} 80 \mathrm{~A}$ & 109.5 & $\mathrm{C} 49-\mathrm{C} 46-\mathrm{C} 48$ & 107.92 (19) \\
\hline $\mathrm{C} 79-\mathrm{C} 80-\mathrm{H} 80 \mathrm{~B}$ & 109.5 & $\mathrm{C} 47-\mathrm{C} 46-\mathrm{C} 37$ & $111.10(18)$ \\
\hline $\mathrm{H} 80 \mathrm{~A}-\mathrm{C} 80-\mathrm{H} 80 \mathrm{~B}$ & 109.5 & $\mathrm{C} 49-\mathrm{C} 46-\mathrm{C} 37$ & $111.75(18)$ \\
\hline $\mathrm{C} 79-\mathrm{C} 80-\mathrm{H} 80 \mathrm{C}$ & 109.5 & $\mathrm{C} 48-\mathrm{C} 46-\mathrm{C} 37$ & $108.60(18)$ \\
\hline $\mathrm{H} 80 \mathrm{~A}-\mathrm{C} 80-\mathrm{H} 80 \mathrm{C}$ & 109.5 & $\mathrm{C} 46-\mathrm{C} 47-\mathrm{H} 47 \mathrm{~A}$ & 109.5 \\
\hline $\mathrm{H} 80 \mathrm{~B}-\mathrm{C} 80-\mathrm{H} 80 \mathrm{C}$ & 109.5 & $\mathrm{C} 46-\mathrm{C} 47-\mathrm{H} 47 \mathrm{~B}$ & 109.5 \\
\hline $\mathrm{C} 78-\mathrm{O} 11-\mathrm{C} 79$ & $118.4(4)$ & $\mathrm{H} 47 \mathrm{~A}-\mathrm{C} 47-\mathrm{H} 47 \mathrm{~B}$ & 109.5 \\
\hline $\mathrm{O} 7-\mathrm{C} 1-\mathrm{Rh} 1$ & $177.5(3)$ & $\mathrm{C} 46-\mathrm{C} 47-\mathrm{H} 47 \mathrm{C}$ & 109.5 \\
\hline $\mathrm{O} 8-\mathrm{C} 2-\mathrm{Rh} 1$ & $179.4(2)$ & $\mathrm{H} 47 \mathrm{~A}-\mathrm{C} 47-\mathrm{H} 47 \mathrm{C}$ & 109.5 \\
\hline $\mathrm{O} 1-\mathrm{C} 3-\mathrm{C} 11$ & $106.33(15)$ & $\mathrm{H} 47 \mathrm{~B}-\mathrm{C} 47-\mathrm{H} 47 \mathrm{C}$ & 109.5 \\
\hline $\mathrm{O} 1-\mathrm{C} 3-\mathrm{C} 5$ & $106.10(14)$ & $\mathrm{C} 46-\mathrm{C} 48-\mathrm{H} 48 \mathrm{~A}$ & 109.5 \\
\hline $\mathrm{C} 11-\mathrm{C} 3-\mathrm{C} 5$ & $112.30(15)$ & $\mathrm{C} 46-\mathrm{C} 48-\mathrm{H} 48 \mathrm{~B}$ & 109.5 \\
\hline $\mathrm{O} 1-\mathrm{C} 3-\mathrm{C} 4$ & $101.22(13)$ & $\mathrm{H} 48 \mathrm{~A}-\mathrm{C} 48-\mathrm{H} 48 \mathrm{~B}$ & 109.5 \\
\hline $\mathrm{C} 11-\mathrm{C} 3-\mathrm{C} 4$ & $112.02(15)$ & $\mathrm{C} 46-\mathrm{C} 48-\mathrm{H} 48 \mathrm{C}$ & 109.5 \\
\hline $\mathrm{C} 5-\mathrm{C} 3-\mathrm{C} 4$ & $117.43(16)$ & $\mathrm{H} 48 \mathrm{~A}-\mathrm{C} 48-\mathrm{H} 48 \mathrm{C}$ & 109.5 \\
\hline $\mathrm{O} 2-\mathrm{C} 4-\mathrm{C} 23$ & $107.17(16)$ & $\mathrm{H} 48 \mathrm{~B}-\mathrm{C} 48-\mathrm{H} 48 \mathrm{C}$ & 109.5 \\
\hline $\mathrm{O} 2-\mathrm{C} 4-\mathrm{C} 17$ & $108.77(14)$ & $\mathrm{C} 46-\mathrm{C} 49-\mathrm{H} 49 \mathrm{~A}$ & 109.5 \\
\hline $\mathrm{C} 23-\mathrm{C} 4-\mathrm{C} 17$ & $106.57(15)$ & $\mathrm{C} 46-\mathrm{C} 49-\mathrm{H} 49 \mathrm{~B}$ & 109.5 \\
\hline $\mathrm{O} 2-\mathrm{C} 4-\mathrm{C} 3$ & $102.67(14)$ & $\mathrm{H} 49 \mathrm{~A}-\mathrm{C} 49-\mathrm{H} 49 \mathrm{~B}$ & 109.5 \\
\hline
\end{tabular}




\begin{tabular}{|c|c|}
\hline $\mathrm{C} 23-\mathrm{C} 4-\mathrm{C} 3$ & $119.38(16)$ \\
\hline $\mathrm{C} 17-\mathrm{C} 4-\mathrm{C} 3$ & $111.80(16)$ \\
\hline $\mathrm{C} 6-\mathrm{C} 5-\mathrm{C} 10$ & $118.05(19)$ \\
\hline $\mathrm{C} 6-\mathrm{C} 5-\mathrm{C} 3$ & $118.22(19)$ \\
\hline $\mathrm{C} 10-\mathrm{C} 5-\mathrm{C} 3$ & $123.49(18)$ \\
\hline $\mathrm{C} 7-\mathrm{C} 6-\mathrm{C} 5$ & $120.7(2)$ \\
\hline $\mathrm{C} 7-\mathrm{C} 6-\mathrm{H} 6$ & 119.6 \\
\hline $\mathrm{C} 5-\mathrm{C} 6-\mathrm{H} 6$ & 119.6 \\
\hline $\mathrm{C} 8-\mathrm{C} 7-\mathrm{C} 6$ & $120.9(2)$ \\
\hline $\mathrm{C} 8-\mathrm{C} 7-\mathrm{H} 7$ & 119.6 \\
\hline $\mathrm{C} 6-\mathrm{C} 7-\mathrm{H} 7$ & 119.6 \\
\hline $\mathrm{C} 7-\mathrm{C} 8-\mathrm{C} 9$ & $118.9(2)$ \\
\hline $\mathrm{C} 7-\mathrm{C} 8-\mathrm{H} 8$ & 120.5 \\
\hline $\mathrm{C} 9-\mathrm{C} 8-\mathrm{H} 8$ & 120.5 \\
\hline $\mathrm{C} 10-\mathrm{C} 9-\mathrm{C} 8$ & $120.6(2)$ \\
\hline $\mathrm{C} 10-\mathrm{C} 9-\mathrm{H} 9$ & 119.7 \\
\hline $\mathrm{C} 8-\mathrm{C} 9-\mathrm{H} 9$ & 119.7 \\
\hline $\mathrm{C} 9-\mathrm{C} 10-\mathrm{C} 5$ & $120.7(2)$ \\
\hline $\mathrm{C} 9-\mathrm{C} 10-\mathrm{H} 10$ & 119.6 \\
\hline $\mathrm{C} 5-\mathrm{C} 10-\mathrm{H} 10$ & 119.6 \\
\hline $\mathrm{C} 12-\mathrm{C} 11-\mathrm{C} 16$ & $118.27(19)$ \\
\hline $\mathrm{C} 12-\mathrm{C} 11-\mathrm{C} 3$ & $120.83(17)$ \\
\hline $\mathrm{C} 16-\mathrm{C} 11-\mathrm{C} 3$ & $120.76(17)$ \\
\hline $\mathrm{C} 11-\mathrm{C} 12-\mathrm{C} 13$ & $120.4(2)$ \\
\hline $\mathrm{C} 11-\mathrm{C} 12-\mathrm{H} 12$ & 119.8 \\
\hline $\mathrm{C} 13-\mathrm{C} 12-\mathrm{H} 12$ & 119.8 \\
\hline $\mathrm{C} 14-\mathrm{C} 13-\mathrm{C} 12$ & $120.5(2)$ \\
\hline $\mathrm{C} 14-\mathrm{C} 13-\mathrm{H} 13$ & 119.7 \\
\hline $\mathrm{C} 12-\mathrm{C} 13-\mathrm{H} 13$ & 119.7 \\
\hline $\mathrm{C} 15-\mathrm{C} 14-\mathrm{C} 13$ & $119.4(2)$ \\
\hline $\mathrm{C} 15-\mathrm{C} 14-\mathrm{H} 14$ & 120.3 \\
\hline $\mathrm{C} 13-\mathrm{C} 14-\mathrm{H} 14$ & 120.3 \\
\hline $\mathrm{C} 14-\mathrm{C} 15-\mathrm{C} 16$ & $120.5(2)$ \\
\hline $\mathrm{C} 14-\mathrm{C} 15-\mathrm{H} 15$ & 119.8 \\
\hline $\mathrm{C} 16-\mathrm{C} 15-\mathrm{H} 15$ & 119.8 \\
\hline $\mathrm{C} 15-\mathrm{C} 16-\mathrm{C} 11$ & $120.9(2)$ \\
\hline $\mathrm{C} 15-\mathrm{C} 16-\mathrm{H} 16$ & 119.6 \\
\hline $\mathrm{C} 11-\mathrm{C} 16-\mathrm{H} 16$ & 119.6 \\
\hline $\mathrm{C} 22-\mathrm{C} 17-\mathrm{C} 18$ & $117.48(19)$ \\
\hline $\mathrm{C} 22-\mathrm{C} 17-\mathrm{C} 4$ & $121.93(16)$ \\
\hline $\mathrm{C} 18-\mathrm{C} 17-\mathrm{C} 4$ & $120.58(15)$ \\
\hline $\mathrm{C} 19-\mathrm{C} 18-\mathrm{C} 17$ & $121.18(18)$ \\
\hline $\mathrm{C} 19-\mathrm{C} 18-\mathrm{H} 18$ & 119.4 \\
\hline $\mathrm{C} 17-\mathrm{C} 18-\mathrm{H} 18$ & 119.4 \\
\hline $\mathrm{C} 20-\mathrm{C} 19-\mathrm{C} 18$ & $120.6(2)$ \\
\hline $\mathrm{C} 20-\mathrm{C} 19-\mathrm{H} 19$ & 119.7 \\
\hline C18-C19-H19 & 119.7 \\
\hline & \\
\hline
\end{tabular}

\begin{tabular}{|c|c|}
\hline $\mathrm{C} 46-\mathrm{C} 49-\mathrm{H} 49 \mathrm{C}$ & 109.5 \\
\hline $\mathrm{H} 49 \mathrm{~A}-\mathrm{C} 49-\mathrm{H} 49 \mathrm{C}$ & 109.5 \\
\hline $\mathrm{H} 49 \mathrm{~B}-\mathrm{C} 49-\mathrm{H} 49 \mathrm{C}$ & 109.5 \\
\hline $\mathrm{O} 10-\mathrm{C} 50-\mathrm{H} 50 \mathrm{~A}$ & 109.5 \\
\hline $\mathrm{O} 10-\mathrm{C} 50-\mathrm{H} 50 \mathrm{~B}$ & 109.5 \\
\hline $\mathrm{H} 50 \mathrm{~A}-\mathrm{C} 50-\mathrm{H} 50 \mathrm{~B}$ & 109.5 \\
\hline $\mathrm{O} 10-\mathrm{C} 50-\mathrm{H} 50 \mathrm{C}$ & 109.5 \\
\hline $\mathrm{H} 50 \mathrm{~A}-\mathrm{C} 50-\mathrm{H} 50 \mathrm{C}$ & 109.5 \\
\hline $\mathrm{H} 50 \mathrm{~B}-\mathrm{C} 50-\mathrm{H} 50 \mathrm{C}$ & 109.5 \\
\hline $\mathrm{O} 5-\mathrm{C} 51-\mathrm{C} 53$ & $107.98(15)$ \\
\hline $\mathrm{O} 5-\mathrm{C} 51-\mathrm{C} 59$ & $104.95(16)$ \\
\hline $\mathrm{C} 53-\mathrm{C} 51-\mathrm{C} 59$ & $111.58(17)$ \\
\hline $\mathrm{O} 5-\mathrm{C} 51-\mathrm{C} 52$ & $103.82(14)$ \\
\hline $\mathrm{C} 53-\mathrm{C} 51-\mathrm{C} 52$ & $113.35(17)$ \\
\hline $\mathrm{C} 59-\mathrm{C} 51-\mathrm{C} 52$ & $114.28(16)$ \\
\hline $\mathrm{O} 6-\mathrm{C} 52-\mathrm{C} 71$ & $106.68(16)$ \\
\hline $\mathrm{O} 6-\mathrm{C} 52-\mathrm{C} 65$ & $106.17(15)$ \\
\hline $\mathrm{C} 71-\mathrm{C} 52-\mathrm{C} 65$ & $111.83(17)$ \\
\hline $\mathrm{O} 6-\mathrm{C} 52-\mathrm{C} 51$ & $104.14(14)$ \\
\hline $\mathrm{C} 71-\mathrm{C} 52-\mathrm{C} 51$ & $113.51(16)$ \\
\hline $\mathrm{C} 65-\mathrm{C} 52-\mathrm{C} 51$ & $113.65(16)$ \\
\hline $\mathrm{C} 54-\mathrm{C} 53-\mathrm{C} 58$ & $117.7(2)$ \\
\hline $\mathrm{C} 54-\mathrm{C} 53-\mathrm{C} 51$ & $120.52(18)$ \\
\hline $\mathrm{C} 58-\mathrm{C} 53-\mathrm{C} 51$ & $121.69(17)$ \\
\hline $\mathrm{C} 55-\mathrm{C} 54-\mathrm{C} 53$ & $120.6(2)$ \\
\hline $\mathrm{C} 55-\mathrm{C} 54-\mathrm{H} 54$ & 119.7 \\
\hline $\mathrm{C} 53-\mathrm{C} 54-\mathrm{H} 54$ & 119.7 \\
\hline $\mathrm{C} 54-\mathrm{C} 55-\mathrm{C} 56$ & $120.8(2)$ \\
\hline $\mathrm{C} 54-\mathrm{C} 55-\mathrm{H} 55$ & 119.6 \\
\hline $\mathrm{C} 56-\mathrm{C} 55-\mathrm{H} 55$ & 119.6 \\
\hline $\mathrm{C} 57-\mathrm{C} 56-\mathrm{C} 55$ & $119.3(3)$ \\
\hline $\mathrm{C} 57-\mathrm{C} 56-\mathrm{H} 56$ & 120.3 \\
\hline $\mathrm{C} 55-\mathrm{C} 56-\mathrm{H} 56$ & 120.3 \\
\hline $\mathrm{C} 56-\mathrm{C} 57-\mathrm{C} 58$ & $119.6(3)$ \\
\hline $\mathrm{C} 56-\mathrm{C} 57-\mathrm{H} 57$ & 120.2 \\
\hline $\mathrm{C} 58-\mathrm{C} 57-\mathrm{H} 57$ & 120.2 \\
\hline $\mathrm{C} 57-\mathrm{C} 58-\mathrm{C} 53$ & $121.9(2)$ \\
\hline $\mathrm{C} 57-\mathrm{C} 58-\mathrm{H} 58$ & 119.0 \\
\hline $\mathrm{C} 53-\mathrm{C} 58-\mathrm{H} 58$ & 119.0 \\
\hline $\mathrm{C} 64-\mathrm{C} 59-\mathrm{C} 60$ & $117.9(2)$ \\
\hline $\mathrm{C} 64-\mathrm{C} 59-\mathrm{C} 51$ & $119.84(18)$ \\
\hline $\mathrm{C} 60-\mathrm{C} 59-\mathrm{C} 51$ & $122.2(2)$ \\
\hline $\mathrm{C} 61-\mathrm{C} 60-\mathrm{C} 59$ & $120.8(2)$ \\
\hline $\mathrm{C} 61-\mathrm{C} 60-\mathrm{H} 60$ & 119.6 \\
\hline $\mathrm{C} 59-\mathrm{C} 60-\mathrm{H} 60$ & 119.6 \\
\hline $\mathrm{C} 62-\mathrm{C} 61-\mathrm{C} 60$ & $120.4(2)$ \\
\hline $\mathrm{C} 62-\mathrm{C} 61-\mathrm{H} 61$ & 119.8 \\
\hline $\mathrm{C} 60-\mathrm{C} 61-\mathrm{H} 61$ & 119.8 \\
\hline
\end{tabular}




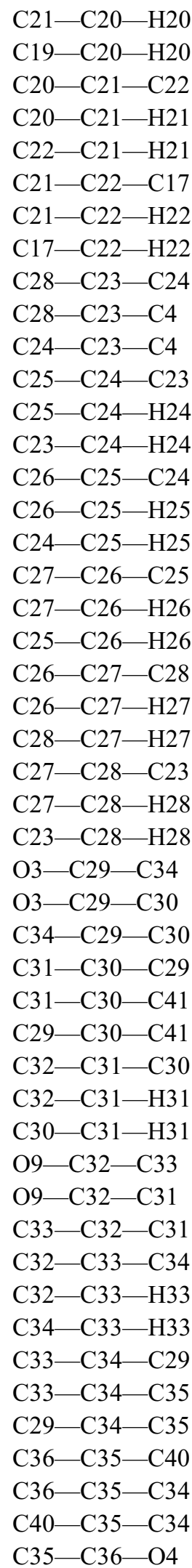

120.6

120.6

$120.8(2)$

119.6

119.6

$121.1(2)$

119.5

119.5

$117.27(19)$

$117.01(18)$

$125.52(18)$

$121.3(2)$

119.3

119.3

$120.5(2)$

119.8

119.8

$119.0(2)$

120.5

120.5

$120.2(2)$

119.9

119.9

$121.7(2)$

119.2

119.2

$117.88(16)$

$120.28(16)$

$121.78(17)$

$116.15(16)$

$119.61(18)$

$123.87(18)$

$122.95(18)$

118.5

118.5

124.77 (19)

115.64 (17)

119.53 (18)

119.48 (18)

120.3

120.3

120.07 (17)

$118.81(17)$

120.77 (17)

$119.96(17)$

$122.90(17)$

$117.05(17)$

$119.71(16)$
C61-C62-C63

C61- C62-H62

C63-C62-H62

C64-C63-C62

C64-C63-H63

C62- $663-\mathrm{H} 63$

C63-C64-C59

C63-C64-H64

C59- C64-H64

C70- $665-\mathrm{C} 66$

$\mathrm{C} 70-\mathrm{C} 65-\mathrm{C} 52$

C66- C65-C52

C67-C66- 65

C67-C66-H66

C65-C66-H66

C68-C67-C66

C68-C67-H67

C66-C67-H67

C69- $668-\mathrm{C} 67$

C69- $668-\mathrm{H} 68$

C67- $668-\mathrm{H} 68$

$\mathrm{C} 68-\mathrm{C} 69-\mathrm{C} 70$

C68- C69-H69

C70- $669-\mathrm{H} 69$

$\mathrm{C} 69-\mathrm{C} 70-\mathrm{C} 65$

$\mathrm{C} 69-\mathrm{C} 70-\mathrm{H} 70$

C65-C70-H70

C76-C71-C72

C76-C71-C52

$\mathrm{C} 72-\mathrm{C} 71-\mathrm{C} 52$

$\mathrm{C} 73-\mathrm{C} 72-\mathrm{C} 71$

$\mathrm{C} 73-\mathrm{C} 72-\mathrm{H} 72$

$\mathrm{C} 71-\mathrm{C} 72-\mathrm{H} 72$

C74-C73-C72

$\mathrm{C} 74-\mathrm{C} 73-\mathrm{H} 73$

C72-C73-H73

$\mathrm{C} 73-\mathrm{C} 74-\mathrm{C} 75$

$\mathrm{C} 73-\mathrm{C} 74-\mathrm{H} 74$

C75-C74-H74

C76-C75-C74

$\mathrm{C} 76-\mathrm{C} 75-\mathrm{H} 75$

$\mathrm{C} 74-\mathrm{C} 75-\mathrm{H} 75$

C75-C76-C71

$\mathrm{C} 75-\mathrm{C} 76-\mathrm{H} 76$

C71-C76-H76

C3-O1-P1

C4-O2-P1

$\mathrm{C} 29-\mathrm{O} 3-\mathrm{P} 1$
119.3 (2)

120.4

120.4

120.1 (2)

120.0

120.0

121.3 (2)

119.3

119.3

$118.1(2)$

$121.6(2)$

$120.26(19)$

$121.2(2)$

119.4

119.4

120.2 (3)

119.9

119.9

119.1 (2)

120.5

120.5

120.6 (3)

119.7

119.7

120.8 (2)

119.6

119.6

118.1 (2)

120.57 (19)

121.4 (2)

120.6 (2)

119.7

119.7

120.5 (2)

119.8

119.8

119.0 (2)

120.5

120.5

120.7 (3)

119.6

119.6

120.9 (2)

119.5

119.5

115.21 (11)

115.76 (11)

121.75 (12) 


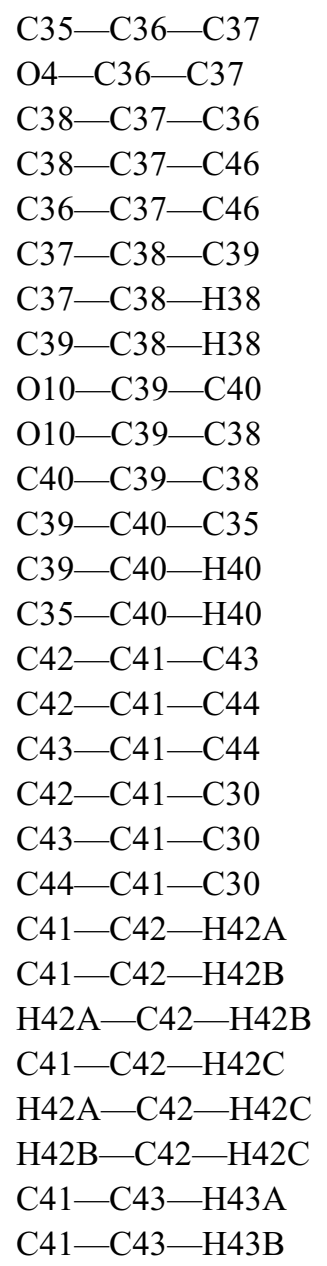

$121.26(18)$

$118.72(17)$

$117.00(18)$

$119.60(18)$

$123.37(18)$

$122.65(18)$

118.7

118.7

$124.98(18)$

$115.50(17)$

$119.52(18)$

$119.39(18)$

120.3

120.3

$109.0(2)$

$108.3(2)$

$106.5(2)$

$107.4(2)$

$114.37(17)$

$111.23(19)$

109.5

109.5

109.5

109.5

109.5

109.5

109.5

109.5

$\begin{array}{ll}\text { C36-O4-P2 } & 129.38(13) \\ \text { C51-O5-P2 } & 118.22(12) \\ \text { C52-O6-P2 } & 118.46(12) \\ \text { C32-O9-C45 } & 117.47(17) \\ \text { C39-O10-C50 } & 117.12(16) \\ \text { O1-P1-O2 } & 93.35(7) \\ \text { O1-P1-O3 } & 101.81(7) \\ \text { O2-P1-O3 } & 101.83(7) \\ \text { O1-P1-Rh1 } & 112.62(5) \\ \text { O2-P1-Rh1 } & 126.26(5) \\ \text { O3-P1-Rh1 } & 116.40(5) \\ \text { O6-P2-O5 } & 94.75(7) \\ \text { O6-P2-O4 } & 105.15(8) \\ \text { O5-P2-O4 } & 98.27(7) \\ \text { O6-P2-Rh1 } & 118.05(6) \\ \text { O5-P2-Rh1 } & 114.74(6) \\ \text { O4-P2-Rh1 } & 121.17(5) \\ \text { C1-Rh1-C2 } & 127.99(13) \\ \text { C1-Rh1-P2 } & 95.80(9) \\ \text { C2-Rh1-P2 } & 89.60(7) \\ \text { C1-Rh1-P1 } & 108.58(9) \\ \text { C2-Rh1-P1 } & 117.99(9) \\ \text { P2-Rh1-P1 } & 109.662(19) \\ \text { C1-Rh1-H1 } & 80.9(14) \\ \text { C2-Rh1-H1 } & 86.9(10) \\ \text { P2-Rh1-H1 } & 172.1(14) \\ \text { P1-Rh1-H1 } & 78.3(14) \\ & \end{array}$

